A N N A L E S Annales de Bretagne et des Pays de l'Ouest

Anjou. Maine. Poitou-Charente. Touraine

125-3 | 2018

Saint-Malo, construction d'un pôle marchand

$(1500-1660)$

\title{
Deux Frondes, un commerce : Rouen et Saint-Malo au milieu du XVII ${ }^{\mathrm{e}}$ siècle
}

Two Frondes, one trade: Rouen and Saint-Malo in the mid-17 th century

\section{Baptiste Etienne}

\section{(2) OpenEdition \\ Journals}

Édition électronique

URL : http://journals.openedition.org/abpo/3925

DOI : 10.4000/abpo.3925

ISBN : 978-2-7535-7691-9

ISSN : 2108-6443

\section{Éditeur}

Presses universitaires de Rennes

Édition imprimée

Date de publication : 6 décembre 2018

Pagination : 79-105

ISBN : 978-2-7535-7572-1

ISSN : 0399-0826

\section{Référence électronique}

Baptiste Etienne, «Deux Frondes, un commerce : Rouen et Saint-Malo au milieu du xvı e siècle »,

Annales de Bretagne et des Pays de l'Ouest [En ligne], 125-3 | 2018, mis en ligne le 06 décembre 2020, consulté le 06 janvier 2021. URL : http://journals.openedition.org/abpo/3925; DOI : https://doi.org/ $10.4000 / a b p o .3925$ 


\title{
Deux Frondes, un commerce : Rouen et Saint-Malo au milieu du XVII ${ }^{\mathrm{e}}$ siècle
}

\author{
Baptiste ETIENNE \\ Doctorant, CRHQ-université de Caen et GRHIS-université de Rouen
}

Au milieu du XVII ${ }^{\mathrm{e}}$ siècle, Rouen est encore la seconde ville du royaume de France et le véritable poumon économique de Paris. Dans le même temps, si Saint-Malo peut être considéré comme " un grand port ${ }^{1}$ ", il demeure une différence d'échelle indiscutable entre ces deux villes ports. Au début du règne de Louis XIV, la ville bretonne comptait entre 15000 et 16000 âmes pour environ cinq fois plus à Rouen². André Lespagnol établit que, contrairement à la métropole normande, Saint-Malo ne constitue pas un marché urbain de première importance, sans qu'il faille toutefois conclure hâtivement à " une faiblesse du pôle capitaliste malouin ${ }^{3}$ ". Par ailleurs, port de fond d'estuaire, Rouen joue aussi un rôle économique, administratif, judiciaire et religieux central, alors que Saint-Malo est situé directement sur le littoral, avec une tradition de quasi "république maritime $^{4}$ ". Fort de deux cents familles d'" entrepreneurs " qui impulsent les activités marchandes et d'une multitude de bailleurs de fonds, à la fin du siècle, Saint-Malo est indéniablement un port d'envergure pour le royaume

1. Pour la fin du XVII ${ }^{\mathrm{e}}$ siècle, on peut considérer qu'avec un tonnage total compris entre 80000 et 100000 tonnes Saint-Malo se situe à une place honorable par rapport à Rouen (LANGLET, Philippe, "Les principaux courants commerciaux du port de Saint-Malo en 1681 et 1682 ", Annales de Bretagne, t. 64, n 3, 1957, p. 323-324; LESPAGNol, André, Messieurs de Saint-Malo - Une élite négociante au temps de Louis XIV, Saint-Malo, L'Ancre de Marine, 1990, p. 172 et CHALINE, Olivier, "La société urbaine des ports de commerce français du Ponant ", dans Poussou, Jean-Pierre (dir.), Les sociétés urbaines au XVII siècle-Angleterre, France, Espagne, Paris, Presses Universitaires de Paris-Sorbonne, 2007, p. 5).

2. Saint-Malo se placerait alors parmi les vingt plus grandes villes du royaume, bien loin des métropoles régionales telles que Nantes, Bordeaux ou Marseille. Pour le milieu du XVII ${ }^{\mathrm{e}}$ siècle, l'estimation du nombre d'habitants pour Rouen oscille entre 75000 et 90000 habitants (LeSPAGNOL André, Messieurs de..., op. cit., p. 38 et BARDET, Jean-Pierre, Rouen aux XVIT et XVII ${ }^{e}$ siècles - Les mutations d'un espace social, t. II, Paris, SEDES, 1983, p. 34-35).

3. Lespagnol, André, Messieurs de..., op. cit., p. 69.

4. Chaline, Olivier, "La société... ", art. cit., p. 5. 
de France ${ }^{5}$. Dans le même temps, Rouen peut compter sur une population marchande de 600 individus, dont la moitié d'étrangers ${ }^{6}$. En outre, jusqu'aux événements politiques de la Fronde, on observe l'établissement de dynamiques en miroir inversé entre ces deux villes portuaires. De plus, si Rouen perd peu à peu de sa vivacité commerciale dans la seconde moitié du XVII ${ }^{\mathrm{e}}$ siècle, Saint-Malo progresse, notamment grâce à la traite négrière et à la course en temps de guerre ${ }^{7}$. Il semble donc complexe de prétendre réaliser une comparaison entre ces deux villes puisqu'elle n'aurait que peu de sens et un intérêt historique limité. Il s'agit bien plus d'étudier la place des Malouins à Rouen par l'intermédiaire de sources presque exclusivement normandes.

La chronologie choisie pour cette recherche s'étend sur l'ensemble du siècle. Toutefois, l'angle politique - point de départ de l'analyse et cœur des problématiques abordées concernant les liens entre Rouen et Saint-Malo invite à mettre l'accent sur la période de la Fronde. Cette révolte trouble la vie politique du royaume de France de 1648 à 1653, lors de la minorité de Louis XIV. On peut légitimement parler de plusieurs Frondes puisque la multiplicité des acteurs - officiers, aristocrates, nobles, peuples - et des causes - impositions, guerre extérieure, mauvaises récoltes - laissent une impression de grande diversité. La Fronde vise avant tout à déstabiliser la régence d'Anne d'Autriche et le principal ministre d'origine italienne, le cardinal Mazarin. Elle touche l'ensemble du royaume de France et cela même si les provinces se sont mobilisées de manière inégale. L'étude de cette révolte ouvre nécessairement de vastes questionnements. La Fronde s'est vue tour à tour qualifiée de diverses façons par les historiens et les érudits qui ont travaillé sur le sujet, allant du " jeu ridicule " de Voltaire jusqu'à la "révolution manquée " proposée par Louis Madelin ${ }^{8}$. À partir des années 1960 , on constate un véritable tournant historiographique puisque la com-

5. Olivier Chaline relève qu'à Rouen, la juridiction consulaire estime que le nombre de marchands reconnus comme " fréquentant la place " s'élève à 520 en 1600 et à 700 en 1656 (Ibid., p. 17 et Lespagnol, André, Messieurs de..., op. cit., p. 39 et 76).

6. Estimation réalisée par Bertrand Gautier dans sa thèse sur le monde marchand de Rouen et de Bordeaux (BotTin, Jacques et Lespagnol, André, " Un couple improbable entre concurrence et coopération : Rouen et Saint-Malo aux Temps modernes ", dans BRIAN, Isabelle (dir.), Le lieu et le moment-Mélanges offerts à Alain Cabantous, Paris, Publications de la Sorbonne, 2015, p. 42 et GAUTIER, Bertrand, Le monde du négoce dans les ports du Ponant sous Richelieu et Mazarin (vers 1625-vers 1660) - Les exemples de Bordeaux et de Rouen, thèse de l'université de Bordeaux III, 1996).

7. À Rouen, à partir de 1687 , la baisse du nombre de marchands serait de près de $25 \%$ en une trentaine d'années (CHALINE, Olivier, "La société... ", op. cit., p. 17; RomAN, Alain, Saint-Malo au temps des négriers, Paris, Karthala, 2003, p. 23 et LeSPAGNOL, André, La course malouine au temps de Louis XIV-entre l'argent et la gloire, Rennes, Apogée, coll. "Hommes et Lieux de Bretagne", 1995, p. 23).

8. GIUZO, Antonella, «Histoire d'une Révolution fille de l'Histoire. Les interprétations de la Fronde au cours de la Révolution française ", dans BouRdin, Philippe (dir.), La Révolution (1789-1871) : écriture d'une histoire immédiate, coll. " histoires croisées ", Presses universitaires Blaise-Pascal, 2008, p. 57-74; VolTAIRE, Lettres philosophiques, Paris, Garnier Frères, 1964, p. 34 et Madelin, Louis, La Fronde - Une Révolution manquée, Paris, Plon, 1931. 
préhension de ce conflit s'ancre dans une célèbre polémique - influencée par des idéologies antagonistes - au sujet de l'interprétation générale des révoltes françaises. D'une possible société bourgeoise en révolte pour Boris Porchnev, à un monde en passe de devenir aristocratique en raison des rapports de patronage dépassant largement les logiques de classes selon Roland Mousnier, les études qui tentent d'analyser ce mouvement complexe se sont enchaînées ${ }^{9}$. Plus récemment, à la faveur des travaux de l'américain Orest Ranum et de Michel Pernot, l'interprétation dialectique de la Fronde a radicalement évolué. L'idée est, désormais, de comprendre cette révolte comme un processus révélant des facteurs de mutations et d'évolutions globales de la société française du Grand Siècle ${ }^{10}$. L'étude économique, en lien avec les bouleversements politiques, prend dès lors une autre dimension puisqu'elle invite à comprendre cette révolte comme le symptôme d'un éventuel tournant économique rouennais.

S'intéresser à la place des Malouins dans la ville de Rouen invite à diversifier les échelles et les points de vue. Du politique au monde marchand, de l'étude de cas de lettres d'affaires à l'analyse des flux internationaux, cette recherche vise à établir dans un premier temps l'importance du lien politique et économique entre ces deux places. Dans un second temps, à travers un exemple précis, il convient de s'interroger sur le rôle joué par le port de Saint-Malo dans le grand commerce rouennais. Afin de réaliser cette étude, un large corpus documentaire normand a été mobilisé. Il s'agit de sources de tout type, institutionnelles (cour des Aides, chambre des Comptes, parlement, juridiction consulaire, etc.) jusqu'à celles du for privé (livres de raison, correspondances, journaux, etc.), qu'il s'agisse de manuscrits ou d'imprimés. Toutefois, peu de documents sont finalement utiles, en raison d'une relative absence de Malouins dans les sources rouennaises.

\section{De la politique au commerce : la place de la Bretagne à Rouen}

\section{Le mythe de l'association politique}

"Outre le voisinage de la Cour, [la Normandie] est tres Importante a cause de sa Richesse, de la mair qui lenvironne, et des fortes plasses qui y sont, et qui semblent de tres aseurees retretes contre tous les acsidens qui peuvent ariver, le voisinage de la bretagne qui est entre les mains dune personne tres afectionet, la doit encor rendre plus considerable a Votre Eminense, ces deus provinsses bien unies ensemble, seroist capables de beaucoup ${ }^{11}$."

9. Porchnev, Boris, Les soulèvements populaires en France au XVIT siècle, Flammarion, Paris, 1970, p. 581 et Mousnier, Roland, La Plume, la faucille et le marteau, Presses Universitaires de France, Paris, 1972, p. 87.

10. Pernot, Michel, La Fronde, Paris, Fallois, 1994 et Ranum, Orest, La Fronde, coll. "L'Univers Historique ", New York, Seuil, 1995.

11. Arch. nat., KK 1083, Recueil Des lettres et memoires concernans la Normandie, fo 118 , lettre de Bougy, 5 mars 1649. 
Cette lettre de Jean Révérend, sieur de Bougy ${ }^{12}$, montre à quel point la proximité géographique entre la Bretagne et la Normandie est un élément essentiel pour l'autorité royale en période de crise politique. Contrairement à ce que suggère cet agent de Mazarin ${ }^{13}$, la situation entre ces deux places est radicalement opposée en 1649. Comme le souligne Hervé Audrain, "il n'y eut pas de Fronde en Bretagne ${ }^{14}$ ". Ainsi, cette province reste loyale à la Couronne, malgré quelques agitations du parlement de Rennes ${ }^{15}$. Depuis 1643 et la mort du cardinal de Richelieu, le gouvernement de cette province est directement sous l'autorité d'Anne d'Autriche. Celui que Bougy qualifie de " tres afectionet " est sans doute le détenteur réel du pouvoir local, c'està-dire son lieutenant général et gouverneur militaire de Nantes, Charles de La Porte, duc de Meilleraye ${ }^{16}$. La Normandie est quant à elle représentative

12. Jean Révérend (1618-1658), sieur de Bougy. D’une famille protestante normande. Il fait une carrière de militaire, successivement cadet dans un régiment des gardes, cornette, capitaine de chevaux légers, capitaine de camp. Il s'est illustré sous les ordres du maréchal de Gassion, puis, à partir de 1647, se rapproche du cardinal Mazarin. Bougy est récompensé de sa fidélité durant le règne de Louis XIV puisqu'il devient lieutenant général dans les armées de France. Durant la Fronde, il est l'auteur d'une douzaine de lettres adressées à Mazarin, essentiellement sur des sujets militaires. Le comte d'Harcourt le décrit comme " tres digne destre considere " (Arch. nat., KK 1083, Recueil Des lettres et memoires concernans la Normandie, $\mathrm{f}^{\circ}$ 102, lettre du comte d'Harcourt, 20 février 1649; Archives historiques de la Gascogne, t. I, Paris, Champion, 1884, p. 149; BAYLE, Pierre, Dictionnaire historique et critique, Rotterdam, R. Leers, 1697, p. 49 et 50 et REVEREND DU MESNIL, Edmond, Mémoires généalogiques sur la maison Le Révérend, sieurs de Basly, Bougy, Calix, La Comté, Soliers, Marquis de Calonges, vicomtes du Mesnil, en Basse-Normandie d'après les documents authentiques, Lyon, Mougin-Rusand, 1882, p. 22 et 23).

13. Le terme d'" agent ", même s'il n'est pas utilisé dans les sources, semble bien adapté puisque le Dictionnaire de l'Académie affirme que cela se dit " de celuy qui fait les affaires d'un Prince ». Le Dictionnaire Universel d'Antoine Furetière va plus loin, en considérant qu'il s'agit d'une commission. Une trentaine d'agents - véritables espions de l'intérieur - tiennent régulièrement informée l'autorité royale de la situation normande (Dictionnaire de l'Académie Française, Première Édition, Paris, 1694, p. 17 et FurETIÈre, Antoine, Dictionnaire universel - Contenant généralement tous les mots françois tant vieux que modernes et les termes des sciences et des arts, t. I, revue et augmentée par M. Basnage de Bauval, 1702, p. 48).

14. Audrain, Hervé, "La Chambre des comptes de Bretagne au temps de la Fronde ", Annales de Bretagne et des Pays de l'Ouest, t. 108, n 4, 2001, p. 137.

15. Ducrest DE Villeneuve, Émile, "La Fronde en Bretagne ", Annales de la Société Académique de Nantes et du département de la Loire-Inférieure, deuxième semestre, 1866, p. 251-279.

16. Charles de La Porte (1602-1664), marquis de La Meilleraye. Issu d'une famille du Poitou, il est cousin germain du cardinal de Richelieu et a été élevé avec lui. En 1632, il est d'abord lieutenant-général de Bretagne, puis gouverneur de Nantes et grand maître de l'artillerie de France. En 1635, il est nommé lieutenant général des armées du roi, puis, en 1639, Maréchal de France. En 1648, il devient surintendant des Finances. Une mazarinade se moquait de lui en ces termes : "n'est-il pas petit fils d'vn miserable notaire de vilage? ", alors que le cardinal de Retz considérait qu'il "était de tous les hommes le plus bas à la cour " (Advertissement a cochon, evesque de Dol et de Fravde - Par les cuistres de l'Université de Paris, 1649, p. 6; DE LA FonTENELlE de VAUDORE, Armand Désiré, Le Maréchal de La Meilleraye, Seconde édition, Poitiers, Saurin frères, 1839; DE SAINTEMARIE, Anselme et DE SAINTE-Rosalie, Ange, Histoire de la Maison Royale de France, et des grands officiers de la Couronne, t. IV, Paris, Compagnie des Libraires, 1726, p. 619-624; 
de cette France frondeuse, comprenant notamment la ville de Paris et des provinces telles que la Guyenne et la Provence. Comme une mise en abîme du royaume dans son ensemble, ces deux provinces présentent donc deux trajectoires politiques radicalement opposées au milieu du XVII ${ }^{\mathrm{e}}$ siècle.

La chronologie de la Fronde en Normandie peut être prise au sens large. Celle-ci intègre alors l'année 1648 avec la mise en place d'une « Fronde institutionnelle ", caractérisée par quelques tentatives infructueuses d'opposition du parlement de Rouen face à l'autorité royale, et s'achève en 1652 avec le dernier arrêt frondeur émanant de cette cour souveraine. Toutefois, au sens strict, la révolte rouennaise est particulièrement courte dans le temps puisqu'elle repose sur l'engagement, à partir du début du mois de janvier 1649, du gouverneur de la province - le duc de Longueville ${ }^{17}$ - dans le parti frondeur. Depuis la journée des barricades de Paris, celui-ci pensait avoir gagné la sympathie du peuple de la capitale et obtenir le commandement des troupes frondeuses. Néanmoins, le choix de donner la direction des armées au prince de Conti, frère de Condé, et le refus du cardinal Mazarin de lui accorder le gouvernement du Havre-de-Grâce, qui était aux mains de la nièce de Richelieu ${ }^{18}$, poussent Longueville à quitter Paris pour se retirer en Normandie. Le 24 janvier 1649, son entrée dans la ville de Rouen signe le début concret du mouvement de révolte dans la province. À partir de ce moment, les cours souveraines et l'hôtel de ville se rallient

Histoire de la Maison Royale de France, et des grands officiers de la Couronne, t. IX, Paris, Compagnie des Libraires, 1733, p. 172; KeTtERING, Sharon, " Patronage and Politics during the Fronde ", French Historical Studies, vol. 14, n 3, 1986, p. 416 et Musset, Victor-Donatien de, Recherches historiques sur le cardinal de Retz : des portraits, pensées et maximes Extraits de ses Ouvrages, Paris, D. Colas, Arthus Bertrand, Delaunay, 1807, p. 262).

17. Henri II d'Orléans (1595-1663), de la maison d'Orléans-Longueville. Prince de France, pair de France, duc de Longueville et autres lieux. A d'abord été gouverneur de Picardie, puis de Normandie à partir de 1619. Dès 1620, il s'est révolté dans le parti de Marie de Médicis, ce qui lui a valu d'être suspendu quelques mois de ses fonctions. Entre 1637 et 1641, il a mené la campagne en Franche-Comté, dans le Piémont, en Alsace et dans le Palatinat. À partir de 1645, il a dirigé la délégation française lors des pourparlers préliminaires des traités de Westphalie qui marquent le terme de la guerre de Trente Ans (1618-1648). Victor Texier disait de lui qu'il " estoit petit, avoit infiniment d'esprit, toujours pirouettant affable et parlant à tout le monde, il marchoit souvent à pied à Rouen son carosse derriere les gardes, jamais devant luy, son chapeau sous le bras et saluant de costé et d'autre " (BnF, fr. 25 0007, Mémoires, par Victor Texier, fo 13; FoISIL, Madeleine, "Une mort modèle - La mort du duc de Longueville, gouverneur de Normandie (1663) ", Annales de Normandie, hors-série, vol. 1, n 1, 1982, p. 243-251; LEBIGRE, Arlette, La duchesse de Longueville, Perrin, Paris, 2004, p. 62 et MORERI, Louis, Le grand dictionnaire historique ou mélange curieux de l'histoire sacrée et profane, t. v, Paris, Le Mercier, Desaint \& Saillant, Herissant, Boudet, Vincent, Le Prieur, 1759, p. 385).

18. Marie-Madeleine de Vignerot (1604-1675), duchesse d'Aiguillon. Ayant échoué dans plusieurs projets de mariage, elle a été dame d'atour de Marie de Médicis. Après le décès du cardinal, elle a hérité d'une partie de ses biens. Le bas âge de son neveu lui permet d'obtenir la tutelle du gouvernement du Havre de 1624 à 1646 (BONNEAU-AvENANT, Alfred, La Duchesse d'Aiguillon, nièce du cardinal de Richelieu, Sa vie et ses Euvres charitables (1604-1675), Paris, Didier, 1879 (rééd. 2012) et DiEFENDORF, Barbara B., From Penitence to Charity - Pious Women and Catholic Reformation in Paris, Oxford University Press, 2004). 
au duc : les portes de la ville sont fermées, les fidèles de l'autorité royale sont expulsés manu militari et on commence à vendre du sel pour faire face aux dépenses courantes et recruter des gens de guerre. Une partie de la clientèle provinciale du duc de Longueville le suit dans ce mouvement de révolte qui se prolonge jusqu'au premier avril de cette même année et la conclusion de la paix de Saint-Germain-en-Laye avec la régente.

Les années suivantes sont marquées par le voyage du roi à Rouen du 6 au 19 février 1650. Mazarin reprend alors l'idée que ses correspondants lui ont suggérée tout au long des mois précédents. Ainsi, dès janvier 1649, le projet est envisagé, puis repoussé par le cardinal qui donne la priorité au règlement de la situation conflictuelle dans la capitale révoltée. Selon le Mémoire sur les affaires de Normandie, ce "seul sesjour " a pour objectif de marquer les esprits du voisinage normand et en particulier celui de la Bretagne et de l'Angleterre ${ }^{19}$. Les deux années suivantes se caractérisent par une opposition parlementaire, durant laquelle royalistes et frondeurs parviennent tour à tour à prendre le contrôle de cette cour souveraine sans qu'une ligne politique précise se dégage. À travers la lettre de Bougy, émerge nettement l'idée d'une union politique entre les deux provinces. Au cours de toute la période de la Fronde, l'attitude des Bretons est scrutée par les agents envoyés en Normandie ${ }^{20}$ : on s'inquiète des agissements de la noblesse ${ }^{21}$, du parlement de Rennes ${ }^{22}$; on évoque aussi d'éventuels renforts royalistes afin de s'attaquer à la Basse-Normandie, tenue par Matignon ${ }^{23}$, ou encore l'approvisionnement en blé de Paris ${ }^{24}$.

L'autorité royale elle-même cherche à contrôler l'association entre ces deux provinces et utilise le marquis de La Meilleraye - lieutenant-général

19. Arch. nat., KK 1083, Recueil Des lettres et memoires concernans la Normandie, fo 353 , "Memoire sur les affaires de Normandie".

20. La correspondance que les agents ont laissée regroupe - pour la période de la Fronde - 224 documents hétéroclites concernant majoritairement la Normandie. Il s'agit à $90 \%$ de lettres, dont 8 sont codées et 7 sont des copies. Les 17 documents restants sont des Mémoires ou Avis. Parmi cet ensemble, certains sont plus surprenants sur la forme, comme des copies d'arrêts du parlement de Normandie ou encore deux lettres intitulées " Nouvelles de Roüen ", datées de 1652. Ces écrits, souvent manuscrits et adressés directement au cardinal Mazarin ou à ses ministres, offrent un observatoire pratiquement quotidien de la situation en Normandie.

21. Arch. nat., KK 1083, fo 70 , lettre anonyme, 4 février 1649 . L'auteur de cette lettre pourrait être Charles de La Porte.

22. Arch. nat., KK 1083, fo 76, lettre de Talon, 6 février 1649.

23. François Goyon de Matignon (1607-1675), cousin germain du duc de Longueville. A été lieutenant-général des armées du roi en Basse-Normandie, puis est devenu gouverneur de Cherbourg et de Granville en 1638 et 1639 et maître de camp d'un régiment d'infanterie en 1643. Durant la Fronde, ce militaire important dans la Manche, était un fidèle du duc de Longueville (DE SAINTE-MARIE Anselme et DE SAINTE-Rosalie, Ange, Histoire de..., op. cit., t. v, p. 387 et Esmonin, Edmond, Mémoire sur la généralité de Rouen (1665) de Voysin de la Noraye, Librairie Hachette, 1913, p. 66).

24. 1000 tonneaux de blés à destination de Paris et en provenance de la Bretagne sont évoqués par La Marguerie en mars 1650 (Arch. nat., KK 1083, Recueil Des lettres et memoires concernans la Normandie, fos 235 et 238). 
de Bretagne - en Normandie au début de l'année 1650. Il se fait alors expert des questions militaires. Cet envoi n'a rien d'un hasard puisque le marquis a déjà exercé des fonctions dans la province et, à titre d'exemple, a obtenu dès 1638 le commandement d'une troupe de huit cents gens d'armes au Pont-Audemer ${ }^{25}$. Ainsi, la Bretagne - qualifiée de " porte de mer et forses maritimes ${ }^{26} "$ - est évoquée à seize reprises dans la correspondance entre l'autorité royale et ses agents en Normandie, ce qui en fait indéniablement un des acteurs importants de ce conflit. En outre, ce type d'association politique entre ces deux provinces a vu le jour dès 1639, lors de la révolte des Nu-pieds à Rouen :

"Sainct-Malo vous demande, Tomblaine, aussy Grandville.

Portz de mer souverains, voisins de Sainct-Michel,

Les requérant, un jour vous serviront d'azyle.

Avecques Jean Nud-piedz, vostre grand colonel ${ }^{27}$."

Dans son Manifeste, Jean Nu-Pieds - meneur énigmatique de la révolte normande - laisse entendre que Saint-Malo et les autres " portz de mer " de Bretagne pourraient constituer un nouveau foyer d'insurrection en appui de la Normandie. Les "requérants " seraient alors, dans la rhétorique des révoltés normands, les Bretons qui " vous demandent " et proposeraient un asile en vue d'une poursuite éventuelle de la révolte en lien ou en succession de celle débutée en Normandie. Revenons brièvement sur les faits. Le 16 juillet 1639, une émeute éclate à Avranches et entraîne le décès de Charles de Poupinel ${ }^{28}$ et de quelques autres personnes. Dans les jours qui suivent, la rébellion se propage en Basse-Normandie jusque dans les centres urbains. Cette révolte semble s'organiser sous les ordres du "général Jean Nu-Pieds " et autour d'une " armée de souffrance ". Ces troupes armées s'en prennent essentiellement aux " gabelleurs ", " monopoliers " et à tous les agents supposés du fisc. En l'espace de trois mois, le mouvement semble avoir atteint son objectif : la perception des impôts est devenue impossible. Cette révolte est restée célèbre en raison de la répression féroce qui l'a suivie, et cela même si elle a été retardée à cause de la guerre

25. Il est possible que La Meilleraye soit présent occasionnellement en Normandie dès le mois de février 1649 (BnF, fr 18938, Papiers de Séguier - Seditions en Normandie, Et la Punition Qui s'en est faicte de la part du Roy, t. II, fo 46 et Arch. nat., KK 1083, Recueil Des lettres et memoires concernans la Normandie, fos 70,171 et 188).

26. Arch. nat., KK 1083, fo 352, "Memoire sur les affaires de Normandie".

27. FloQuET, Amable, Diaire ou journal du voyage du chancelier Séguier en Normandie après la sédition des Nu-pieds (1639-1640) et documents relatifs à ce voyage et à la sédition, Edouard frère, 1842, p. 408.

28. Charles de Poupinel (?-1639), sieur de la Besnardière, a été lieutenant particulier au bailliage de Coutances. Sur son décès, le Diaire évoque que " [le peuple] pressé d'un désespoire furieux, assaillit le dict Poupinel en pleine rue, et l'assoma cruellement à coup de pierre, de baston et autres armes. Il y avoit pas plus de 40 à 50 coquins, au commencement, la plusparts aulniers et porteurs de bois; mais, en moins d'un quart d'heure, tout le peuple grossit la cohorte, en sorte qu'à la fin, il s'y en trouva plus de 400 " (FoISIL, Madeleine, La Révolte des Nu-Pieds et les révoltes normandes de 1639, Paris, PUF, 1970, p. 175 et Floquet, Amable, Diaire..., op. cit., p. 397 et 398). 
européenne $^{29}$. Encore une fois, on observe une certaine distorsion entre les discours et les faits. L'association entre les deux provinces tient du mythe politique puisque la Bretagne n'a jamais rejoint les rangs des révoltés. Elle n'a pas non plus offert de refuge au " capitaine Va Nuds-Piedz ", comme l'affirme Verthamont ${ }^{30}$ dans sa Relation du voyage du chancelier, suivant par cette remarque l'idée avancée par le Manifeste ${ }^{31}$.

Ainsi, qu'il s'agisse d'une union poussée par l'administration royale afin de permettre un meilleur contrôle du territoire durant la Fronde ou d'un lien fictif supposé, en période de crise, la Cour est régulièrement tentée de faire de ces deux provinces une seule et même entité politique. La volonté de ramener la Normandie vers un apaisement en la rapprochant de sa voisine, plus calme au cours de ces évènements, semble être véritablement au cœur des préoccupations royales. Les contemporains semblent donc voir, en permanence, un lien étroit entre ces deux provinces, si proches géographiquement, mais si éloignées politiquement. Cette association de l'ordre du mythe se retrouve-t-elle dans d'autres domaines à travers les sources rouennaises?

Si on observe en détail la présence bretonne à Rouen, on constate avant tout une certaine absence dans les sources du milieu du XVII ${ }^{\mathrm{e}}$ siècle. En témoigne le peu de Malouins relevés dans les actes de décès rouennais de la génération 1650, alors que dans le même temps on note la présence de près de 800 horsains et étrangers ${ }^{32}$. Ainsi, on ne dénombre que cinq individus originaires de cette ville portuaire, dont un serviteur des messagers entre Rouen et Saint-Malo et un homme de chambre de l'évêque ${ }^{33}$. Les Bretons sont aussi largement sous-représentés puisqu'on en décompte qu'une douzaine supplémentaire, dont deux sont matelots et un bourgeois $^{34}$. Qu'ils soient originaires de Saint-Malo, La Chaîne, Nantes, Rennes,

29. FoIsIL, Madeleine, La révolte..., op. cit., p. 311.

30. François de Verthamont (1597-1666), seigneur et baron de Breau et marquis de Manœuvres. À partir de 1618, il a été conseiller au parlement de Paris puis, en 1626, maître des requêtes ordinaires de l'hôtel du roi. Par la suite, il a été intendant de justice au siège de La Rochelle, puis de l'armée d'Italie et de l'armée de Guyenne de 1630 à 1638. À partir de 1643, il est conseiller d'État (Floquet, Amable, Diaire..., op. cit., p. x).

31. Ibidem, p. 285.

32. Il s'agit des actes de décès des Rouennais nés avant 1648 et décédés avant 1705, ce qui constitue un corpus de près de 50000 individus.

33. Louis Houel (1642-1672) est serviteur de Tillers et Cussy, messagers de Rouen à Saint-Malo. Il est décédé et enterré catholiquement paroisse Saint-Jean. Alors qu'un homme de chambre de Ferdinand de Neufville de Villeroy, évêque de Saint-Malo, âgé de 35 ans, est décédé à Rouen en 1652 et enterré, catholiquement, paroisse Saint-Laurent (Arch. dép. de Seine-Maritime, 3 E 00999, Registre paroissial de Saint-Jean (1668-1673), registre 249, acte du 17 août 1672 et 3 E 00999, Registre paroissial de Saint-Laurent (16241657), registre 270, registre des sépultures, acte du 25 août 1652).

34. Pierre Jouanne, matelot de Bretagne, s'est noyé à Rouen en 1701 alors qu'il naviguait sur la gribane nommée L'Espérance de La Pain et dont le capitaine est le protestant Jean Le Roux. Il est enterré catholiquement à Saint-Maclou. Hervé Perrot est un matelot breton, décédé dans la paroisse Saint-Vincent. Guillaume Benard (1630-1693), bourgeois de Rennes et enterré dans la paroisse de Saint-Candé-Le-Vieux. S'ajoute Louis Sorbecq 
ceux-ci ne sont qu'une infime part du contingent des horsains ${ }^{35}$. La présence rouennaise à Saint-Malo ne paraît pas plus développée, comme en témoigne l'analyse de l'immigration dans ce port breton réalisée par André Lespagnol. Ainsi, si les Normands constituent le principal pourvoyeur de migrants - en dehors de la Bretagne même - ceux-ci sont pratiquement exclusivement issus de la Basse-Normandie voisine ${ }^{36}$. L'ensemble des sources normandes laisse donc à penser qu'au XVII ${ }^{\mathrm{e}}$ siècle, Rouen a un net tropisme vers le bassin parisien et délaisse majoritairement ses liens avec la côte atlantique. Toutefois, d'autres documents dénotent une présence malouine à Rouen. Ainsi, à la fin des années 1640, l'évêque de Saint-Malo ${ }^{37}$, en tant qu'abbé commendataire de Saint-Wandrille, fait valoir ses droits à deux reprises au bureau des finances de la généralité de Rouen. Sa requête vise à recouvrer les sommes de 444 et 630 livres que Nicollas Le Cauchois et Jacques Harel, receveurs du domaine, lui doivent des années précédentes. Quatre jours plus tard, face au refus d'un des receveurs, il est contraint d'effectuer une nouvelle requête ${ }^{38}$. Toutefois, cette affaire est inhabituelle puisque les Malouins sont peu représentés dans les procédures judiciaires à Rouen, mis à part le milieu marchand qui fait figure d'exception notable. Ainsi, ces deux provinces voisines sont régulièrement associées à travers les problématiques commerciales.

\section{Le commerce au cour des liens}

Les liens commerciaux entre Saint-Malo et Rouen sont anciens puisque la première mention d'une arrivée importante de navires malouins dans la capitale normande est évoquée à la fin de la première guerre de Religion. Comme le souligne Jacques Bottin, les échanges entre ces deux ports peuvent sembler médiocres, mais ils reflètent essentiellement une relation de complémentarité qui implique des rapports de collaboration ${ }^{39}$. S'ajoute nécessairement la mise en place de conflits, comme en témoigne ce procès

(?-1691), décédé en tant que forçat à Rouen (Arch. dép. de Seine-Maritime, 3 E 00999, Registre paroissial de Saint-Maclou (1701-1704), registre 418, registre des sépultures, acte du 2 septembre 1701; 3 E 00999, Registre paroissial de Saint-Vincent (1647-1662), registre 633, acte du 8 février 1661; 4 E 01991, Registre paroissial de Saint-Candé-le-Vieux (16761695), acte du 28 juillet 1693 et 3E 00999, Registre paroissial de Saint-Gervais (1691-1699), registre 156 , acte du $1^{\mathrm{er}}$ mars 1691$)$.

35. Six sont issus de La Chaîne, trois de Nantes et respectivement un de Rennes ou de Saint-Brieuc. Les Bretons ne représentent que $2 \%$ des horsains, ce qui équivaut à un peu plus de la moitié des effectifs parisiens.

36. Lespagnol, André, Messieurs de..., op. cit., p. 82-83.

37. Ferdinand de Neufville de Villeroy (1608-1690). A été conseiller d'État d'Église, évêque de Saint-Malo de 1644 à 1657, puis de Chartres de 1657 à 1690 (TELLIER, Luc Normand, Face aux Colbert - Les Le Tellier, Vauban, Turgot... et l'avènement du libéralisme, Presses de l'Université du Québec, 1987, p. 796 et BERgin, Joseph, The Making of the French Espiscopate (1589-1661), New Haven et Londres, Yale University Press, 1996, p. 677).

38. Arch. dép. de Seine-Maritime, C 1153, Plumitifs du bureau des finances de la généralité de Rouen, actes du 14 décembre 1648 et du 18 décembre 1648.

39. Bottin, Jacques et LesPagnol, André, "Un couple... ", art. cit., p. 51. 
de juin 1627, qui oppose un Malouin et un Rouennais devant la juridiction consulaire ${ }^{40}$. S'il s'agit d'un cas isolé, celui-ci est révélateur de l'implication du milieu marchand malouin dans la capitale normande.

" Devant nous Les prieur et consuls des marchands de Rouen Sur Ce que honnorable homme Nicollas Blondel ${ }^{41} \mathrm{p}$ [rése]nt en Personne stippullant pour Le sieur Estienne Goret marchand A sainct Malo avoict faict convenir et adjourner honnorable homme Nicollas de Baucquemare ${ }^{42}$ aussypresent en personne affin de sommer Ou amender ung deffault ${ }^{43}$."

Rares sont les procès impliquant des Rouennais et des Malouins au sein des juridictions normandes. Dans ce cas précis, l'ajournement demandé par Nicolas Bauquemare fait suite à un premier procès touchant le commerce de l'alun et qui s'est déroulé trois ans auparavant. Le Rouennais se sert justement de ce long intervalle pour gagner encore du temps et pour demander que les papiers lui soient à nouveau communiqués directement entre ses mains, ne " pouvan commodement veoir au Greffe ". Blondel rétorque que cette procédure a pour unique objet de régler un ou deux articles supplémentaires, le procès ayant déjà été jugé sur le fond. Néanmoins, les consuls déboutent le représentant du Malouin et ordonnent au greffier de fournir à nouveau les pièces de cette affaire. En août, la procédure rebondit puisque Bauquemare refuse de se faire remettre un formulaire de décharge, arguant être parvenu à casser quelques éléments du procès précédent. À partir de cette époque et jusqu'à la décennie 1650, la famille Bauquemare entreprend un véri-

40. Depuis 1556, Rouen dispose d'une juridiction consulaire à l'image d'autres grandes villes du royaume comme Paris, Lyon ou encore Marseille. Membres de l'administration municipale, les consuls sont chargés de veiller aux intérêts de la navigation, mais ils ont aussi pour objectif de juger les litiges commerciaux et les faillites. Au XVII ${ }^{\mathrm{e}}$ siècle, cette institution est installée rue de l'Estrade et elle est composée d'un procureur-syndic, d'un premier et d'un second consul et prieur, d'une vingtaine de prieurs et d'une quinzaine de consuls des marchands, ainsi que d'un chapelain qu'elle choisit (Arch. dép. de Seine-Maritime, 201 BP 670, Registre des délibérations de l'assemblée des marchands (1648-1678); Lafosse, Henri, La Juridiction Consulaire de Rouen (1556-1791), Rouen, Henri Defontaine, 1922, p. v, 19, 91 et ULBERT, Jörg, " La fonction consulaire à l'époque moderne : définition, état des connaissances et perspectives de recherche ", dans ULBERT, Jörg et LE BOUËDEC, Gérard (dir.), La fonction consulaire à l'époque moderne - L'affirmation d'une institution économique et politique (1500-1800), Rennes, Presses universitaires de Rennes, coll. " Histoire", 2006, p. 10.

41. Il pourrait s'agir de Nicolas Blondel, sieur de Coquetot, marchand. Il aurait tenté de se faire recevoir à la charge de clerc du Parlement. Il est en lien dans ses affaires avec les Pays-Bas (Arch. dép. de Seine-Maritime, 2 E 1 2467, actes du 17 avril 1649, 26 juin 1649 et 10 juillet 1649 et 2 E 12391 , actes du 17 février 1650,14 mars 1650 et 24 avril 1650).

42. Il pourrait s'agir de Nicolas de Bauquemare (1580-1647), bourgeois de Rouen. En 1623, il a épousé Marie Moisant (Arch. dép. de Seine-Maritime, 201 BP 643, Juridiction consulaire, Papiers de Bauquemare, procès-verbal, fo 1 et Frondeville, Henri de, Les présidents du Parlement de Normandie (1499-1790) - Recueil généalogique établi sur la base du manuscrit Bigot de la Bibliothèque à Rouen, Paris et Rouen, A. Lestringant et A. Picard, 1953, p. 57).

43. Arch. dép. de Seine-Maritime, 201 BP 623, Juridiction consulaire, Expédition de sentences (1627-1727), année 1627. 
table marathon devant les consuls des marchands en utilisant de manière constante ce type de méthode de harcèlement judiciaire ${ }^{44}$.

Au milieu du XVII ${ }^{\mathrm{e}}$ siècle, cette juridiction n'est pas uniquement un espace d'affrontement puisque les communautés marchandes rouennaise et malouine peuvent, à l'occasion, trouver un terrain d'entente et des moyens d'action communs. Le 30 octobre 1652, l'assemblée des marchands de Rouen décide la députation de Bertrand Derbland ${ }^{45}$ et de Philippe Beguin ${ }^{46}$, afin d'agir conjointement avec la communauté de Saint-Malo. Les deux juridictions s'unissent afin de réclamer la restitution des marchandises, argent et " autres effects " du navire L'Aurore, alors arrêté à Nantes en exécution d'un arrêt du conseil du roi. On peut supposer que le fret de ce navire était composé d'actifs détenus par des marchands de Rouen et de Saint-Malo, ce qui expliquerait cette association inhabituelle. Au milieu du siècle, ce type d'actes administratifs associant le monde des marchands malouins à celui de Rouen est rare. Seule l'activité notariale offre de réelles perspectives pour l'analyse de la présence malouine en Normandie au milieu du XVII ${ }^{\mathrm{e}}$ siècle ${ }^{47}$.

L'implication des marchands malouins à Rouen est ancienne et a déjà fait l'objet de nombreuses études ${ }^{48}$. En l'espace de trois ans, une dizaine d'actes isolés du tabellionage de Rouen concernent directement des Malouins. Ainsi, en 1660, le marchand rouennais Guillaume Thiboutot ${ }^{49}$

44. Arch. dép. de Seine-Maritime, 201 BP 636, Juridiction consulaire, Papiers de faillis (1641-1650) et 201 BP 637, Juridiction consulaire, Papiers de faillis (1651-1660).

45. L'acte des consuls des marchands inverse les fonctions des deux députés. Bertrand Derbland (1609-1669), marchand et ancien juge consul des marchands. En 1650, il est intéressé à la cargaison d'un navire hollandais avec huit autres marchands rouennais. Afin de veiller sur leurs intérêts, ils nomment comme procureur spécial Jacques Fermanel, sieur de l'Epinay. Derbland est aussi régulièrement en affaires avec le monde ibérique (Arch. dép. de Seine-Maritime, 3E 00999, Registre paroissial de Saint-Nicolas (1631-1674), registre 504, registre des sépultures, acte du 5 juillet 1669; 2 E 12391 , acte du 8 février 1650 et $2^{\mathrm{E}} 12491$, actes du 4 novembre 1660, 6 novembre 1660,15 novembre 1660 et 18 novembre 1660$)$.

46. Philippe Beguin (1601-1672), marchand, juge et prieur des consuls des marchands, puis gouverneur. Il a été administrateur de l'Hôtel-Dieu de Rouen et a donné dans son testament 6000 livres tournois pour la célébration journalière d'une messe basse à l'église paroissiale de Saint-Candé-Le-Jeune, de laquelle il a été trésorier. Dans ses affaires, il semble régulièrement en relation avec les Pays-Bas et l'Espagne (Arch. dép. de SeineMaritime, 3 E 00999, Registre paroissial de Saint-Cande-le-Jeune (1668-1672/02/21), acte du 12 février $1672 ; 2$ E 12490 , actes du $1^{\text {er }}$ avril 1660, 6 avril 1660 et 22 avril 1660 et 2 E 1 2491, actes du 7 juillet 1660, 20 octobre 1660 et 22 octobre 1660).

47. Ne sont pris en compte que les actes notariés des années 1649, 1650 et 1660 . Ce sondage est encore insuffisant pour être réellement représentatif et il est nécessaire qu'à terme, l'ensemble des actes durant la période soit étudié afin d'offrir une vision plus complète de la présence malouine à Rouen.

48. Gosselin, Édouard, Documents authentiques et inédits pour servir à l'histoire de la marine normande et du commerce rouennais pendant les XVI et XVIII siècles, Paris, Henry Boissel, 1876, p. 97 et 166 et Mollat, Michel, Le commerce de la Haute Normandie au $X V^{e}$ siècle et au début du XVI ${ }^{e}$, Paris, Plon, 1952, p. 16, 19, 40, 81, 85.

49. Guillaume Thiboutot aurait épousé Barbe Talon (1622-1688) en 1644 (Arch. dép. de Seine-Maritime, 3 E 00999, Registre paroissial de Saint-Nicaise (1680-1700), registre 
tire une lettre du change, rédigée à Saint-Malo, de 700 livres pour Estienne Troullet. Celle-ci, présentée au banquier Jean Musnier ${ }^{50}$, est finalement protestée par les parties ${ }^{51}$. Deux autres actes touchent la constitution de procureurs spéciaux afin de se faire remettre des fonds. La même année, Théophile Pineau, maître de la monnaie de Rouen, cherche à se faire payer de Noüal ${ }^{52}$, marchand de Saint-Malo. Enfin, en 1649, un acte évoque une affaire complexe commencée en 1643 entre Jacques Rouvray et le Malouin Louis Gravé, sieur des Rochettes. Il s'agit pour le Rouennais d'obtenir - pour le compte de deux autres marchands - le payement de 12288 livres sur deux sacs de cochenille. En 1645, la marchandise avait été refusée à Rouen, par les premiers marchands impliqués dans cette affaire, avant que deux autres prennent le relais. Toutefois, insatisfaits de la qualité de la marchandise, ces derniers obtiennent condamnation de Rouvray et de Gravé à la fin de l'année ${ }^{53}$. Signe de l'importance de ces marchands dans la capitale normande, cela représente plus d'un tiers de l'ensemble des actes des Bretons à Rouen. De ce point de vue, seuls les Nantais semblent être en mesure de concurrencer les marchands de Saint-Malo avec cinq actes, pour trois des Morlaisiens et un seul pour les Rennais. Ces actes malouins font donc écho à cinq autres concernant des marchands bretons.

Comme on le constate sur la carte de répartition par paroisse ${ }^{54}$, les hôtelleries se concentrent au nord de la ville, loin du port. La paroisse Saint-Martin-sur-Renelle ${ }^{55}$ accueille la plus grande concentration de lieux d'accueil. Néanmoins, seuls deux Bretons logent dans les hôtelleries rouennaises : le Gaillard Bois et La Magdellaine ${ }^{56}$. Alors qu'il existe

490, acte du 26 janvier 1688 et $3^{\text {E }}$ 00999, Registre paroissial de Saint-Vincent (1634-1661), registre 632 , acte du 16 mai 1644$)$.

50. Jean Musnier ou Le Munier, avocat au Parlement de Rouen et banquier en cour de Rome. A épousé Marie Le Marié (?-1670) (Arch. dép. de Seine-Maritime, 3 E 00999, Registre paroissial de Saint-Laurent (1668-1680), registre 276, acte du 29 août 1670).

51. Arch. dép. de Seine-Maritime, 2 E 12726 , acte du 10 avril 1660.

52. Il pourrait s'agir de Jacques Nouail (1640-1680), sieur du Fougeray. Toutefois, l'acte rouennais indique qu'il possède la seigneurie de Pomprin. Issu d'une famille de la bourgeoisie marchande de Vitré. Il pourrait être protestant et ne s'installe à Saint-Malo que dans les années 1660 et s'y convertit au catholicisme puisqu'en 1673 il épouse Françoise Cheville, d'une famille de la bourgeoisie catholique locale. Négociant notable, orienté vers le commerce de Cadix. Il pourrait aussi s'agir d'un membre de la famille Nouel. Vieille famille malouine implantée dans la ville dès le XV siècle. Au XVII ${ }^{\mathrm{e}}$ siècle, ils se situaient dans les couches " moyennes " de la bourgeoisie marchande (LESPAGNOL, André, Messieurs de..., op. cit., p. 837).

53. Arch. dép. de Seine-Maritime, 2 E 1 2387, acte du 5 mars 1649.

54. Conception de la carte par les auteurs, avec l'aide - pour la cartographie - de F. Delisle (GRHIS), à partir des plans géoréférencés par G. Gaillard, L. Levieux et B. Etienne, ainsi que la carte des paroisses réalisée par J.-P. Bardet.

55. Durant le XVII ${ }^{\mathrm{e}}$ siècle, il s'agit d'une paroisse en lien avec les métiers du textile, mais également avec le monde des artisans et des officiers. Il s'agit donc d'une des paroisses figurant parmi les plus privilégiées de la ville.

56. Le Grand Gaillard Bois, situé paroisse Saint-Candé-le-Vieux, est en activité au minimum de 1649 à 1660. Sa clientèle est essentiellement constituée d'horsains, à l'image d'Allexandre Bigot, marchand de Rennes. L'auberge de La Madeleine se situe rue des 


\section{Figure 1 - Les hôtelleries à Rouen en 1676}

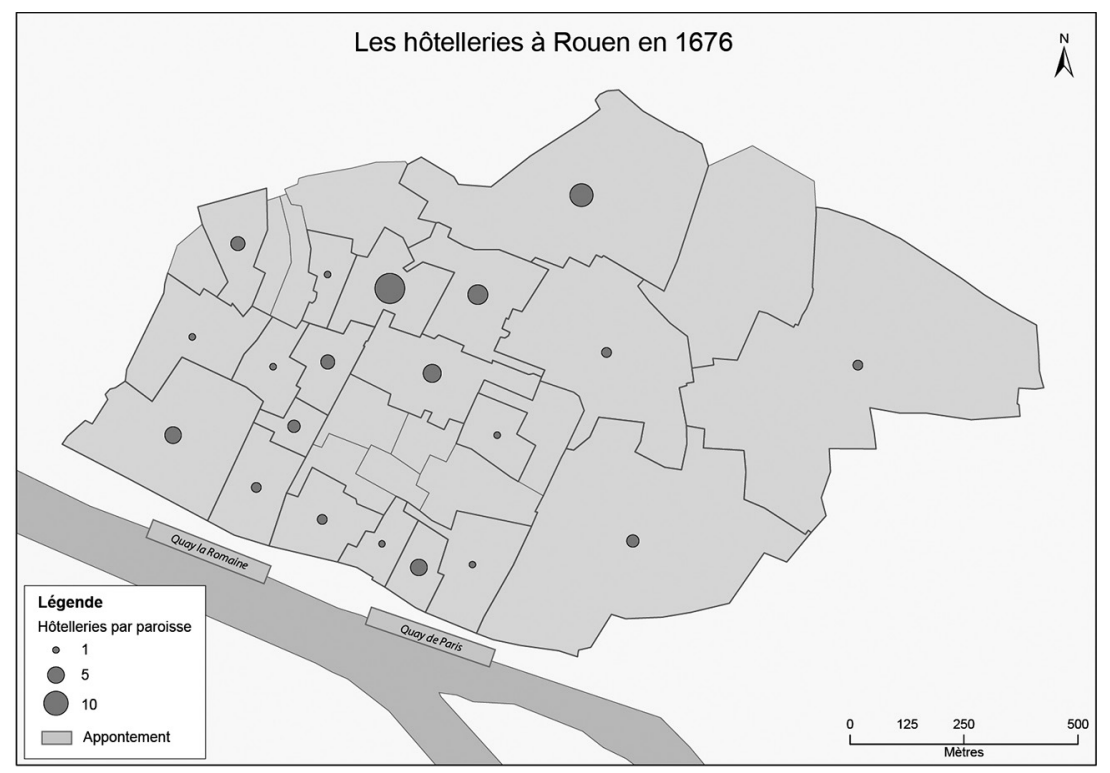

127 auberges actives du milieu du siècle à $1676^{57}$, ces marchands bretons sont des exceptions puisque ces échanges se font majoritairement par l'intermédiaire d'actes de procuration, permettant d'éviter des déplacements. On en trouve d'autres : en 1649, au lendemain de la Fronde, le marchand nantais Noël de Farye épouse Juste de Fonseca. Celle-ci est issue d'une famille originaire du Portugal qui a entrepris d'émigrer à Rouen à partir des années 1610. Lors de son mariage, Juste était naturalisée depuis seulement deux ans. Le registre des naturalisations indique qu'ils avaient l'intention de "passer le reste de leur vye " à Rouen comme le reste de ses parents et cela même si certains se sont installés par la suite sur l'île de Tenerife aux Canaries ou ont fait le choix de rester au Portugal ${ }^{58}$. Du point de vue de la famille Fonseca ${ }^{59}$, cette exogamie géographique résulte de dif-

Belles Femmes, paroisse Saint-André. Celle-ci est tenue en 1650 par la veuve Jeanne Du Busc, qui héberge le marchand Jean Lory, natif de Nantes (Arch. dép. de Seine-Maritime, 2 E 1 2694, acte du 30 août 1649; 2 E 1 2341, acte du 17 septembre 1649; 2 E 1 2392, acte du 2 juillet 1650 et 6 juillet 1650 et 2 E 12705 , acte du 21 septembre 1660 et $1^{\text {er }}$ octobre 1660 ).

57. L'identification de ces hôtelleries a été réalisée grâce aux registres paroissiaux des décès de 1650 à 1705 et aux actes notariés des années 1649, 1650 et 1660 .

58. Arch. dép. de Seine-Maritime, 2 E 1 2694, acte du 4 mars 1649; 2 B 361, Chambre des Comptes, Lettres de naturalité et d'anoblissement (1631-1789), acte du 23 février 1647 et VENDRAND, Céline, Les étrangers résidant au Havre et à Rouen au XVII siècle, t. 2, Caen, master 2, Université de Caen, 2002, p. 99.

59. Cette famille est installée à Rouen depuis le début du XVII ${ }^{\mathrm{e}}$ siècle puisqu'une Louise de Fonseca, originaire de Lisbonne, arrivée à Rouen en 1614, est naturalisée en octobre 1616 et Isabelle de Fonseca, originaire du Portugal, fille de Gonzade Cardoze et de Cecille 
ficultés à Rouen, en raison de leur situation depuis les années 1630 et de l'affaire décrite par Natalia Muchnik ${ }^{60}$. Soupçonnés de cryptojudaïsme, les Fonseca subissent la pression inquisitoriale jusqu'à la capitale normande, sans que l'accusation puisse être prouvée. Dès lors, on comprend que ces naturalisés soient forcés de chercher des mariages hors de la Normandie, ne pouvant se limiter au strict marché matrimonial des émigrés portugais. Ainsi, en 1645, seule Marguerite Fonseca, sœur de Juste, épouse Jacques Henriques qui appartient lui aussi à la communauté portugaise installée à Rouen ${ }^{61}$.

À la différence de ceux concernant les Malouins, une petite partie des actes bretons portent sur d'autres catégories sociales que les marchands. À l'image d'Anne de Boullé, veuve en secondes noces d'un président au parlement de Bretagne, qui est contrainte de verser à Marie Auber ${ }^{62}$ la somme de 5400 livres pour les dettes que son mari a contractées quarante ans auparavant ${ }^{63}$. De ce point de vue, les officiers des cours souveraines sont largement représentés. Ainsi, que ce soit les familles parlementaires des Deshommets en instance devant le parlement de Bretagne, des Du Moucel ou encore des Langlois, trésoriers de France de la généralité de Rouen, tous passent des actes avec des Bretons ${ }^{64}$. Ces derniers - et en particulier les Malouins - sont donc actifs à Rouen et se retrouvent dans de nombreux actes notariés. Tous n'appartiennent pas au monde du commerce, mais cela démontre assurément que l'implication des voisins bretons dépasse largement ce qu'un premier aperçu des sources pouvait laisser penser. D'autres exemples existent, mais c'est probablement celui du Rouennais Robert de La Fosse qui offre une certaine diversité et l'approche la plus concrète du lien entre Saint-Malo et Rouen pour le grand commerce.

Rodrigues, mariée Simon Loppes Manuel, est naturalisée en mars 1630 (Archivo General de Simancas, K 1416, fol. 10, "Note d'Antonio Hurtado de Mendoza », 14 avril 1633; Arch. dép. de Seine-Maritime, 2 B 361, Lettres de naturalité et d'anoblissement (1631-1789), acte du 14 octobre 1614 et acte du 20 mars 1630; 2 E 1 2694, acte du 4 mars 1649; MucHNIK, Natalia, "Du catholicisme des judéoconvers : Rouen, 1633 ", Dix-septième siècle, n² 231, 2006, p. 277-278 et VEndRand, Céline, Les étrangers..., op. cit., p. 99-100).

60. MuCHNIK, Natalia, "Du catholicisme... ", art. cit., p. 277-299.

61. Arch. dép. de Seine-Maritime, 2 B 361, Chambre des Comptes, Lettres de naturalité et d'anoblissement (1631-1789), acte du 15 décembre1623 et VENDRAND, Céline, Les étrangers..., op. cit., p. 36 et 106-109.

62. Arch. dép. de Seine-Maritime, 2 E 1 2296, acte du 11 septembre 1660.

63. Arch. dép. de Seine-Maritime, 2 E 1 2342, acte du 14 avril 1650.

64. La famille des Deshommets est issue du monde des marchands. À partir du milieu du XVI ${ }^{\mathrm{e}}$ siècle, elle s'engage dans l'acquisition d'offices. Les Du Moucel sont issus d'une famille de monnayeurs de Rouen, anoblie en 1544. Les Langlois ont investi dans l'achat d'offices dès le $x^{\mathrm{e}}{ }^{\mathrm{e}}$ siècle, jusqu'à l'entrée au parlement de Bruno-Emmanuel de Motteville en 1692 (Arch. dép. de Seine-Maritime, 2 E 1 2387, acte du 19 janvier 1649; 2 E 1 2467, acte du 7 août 1649; FrondeVILle, Henri de, Les présidents..., op. cit., p. 450, 451 et 477 et Les conseillers du Parlement de Normandie sous Henri IV et sous Louis XIII (1594-1640) - Recueil généalogique établi sur la base du Manuscrit Bigot, de la Bibliothèque de Rouen, $\mathrm{t}$. III, Rouen et Paris, A. Lestringant et Auguste Picard, 1964, p. 171). 


\section{Robert de La Fosse : Rouen, Saint-Malo et le trafic international}

\section{Robert de La Fosse et son réseau}

Robert de La Fosse ${ }^{65}$ est un marchand mercier rouennais qui entretient de solides relations avec Saint-Malo à partir du début des années 1660. On dispose de peu d'éléments sur lui, mis à part qu'il s'est rendu en Italie et aurait été atteint d'une fluxion durant six semaines en 1671. En tant que marchand mercier, son commerce est relativement diversifié puisqu'il s'intéresse aux toiles, charbons, quincailleries, boutons et, entre autres, aux teintures. Il dispose d'une capacité financière importante dans les années 1660; ainsi, en 1668, il annonce avoir perdu depuis six ans environ 60000 livres et 40000 écus. Il nous a laissé un registre comportant des copies de plus de 170 lettres envoyées en 1647 et 1648, puis de 1660 à décembre $1671^{66}$.

Figure 2 - La correspondance de Robert de La Fosse

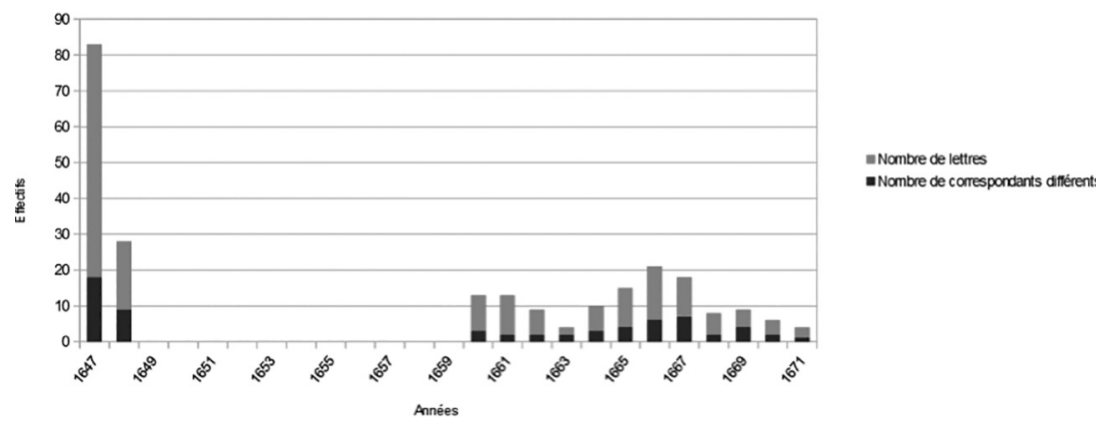

De 1649 à 1659, lors de l'interruption de transcription des lettres, tout laisse à penser que ce marchand a continué à faire des affaires. On peut supposer qu'à partir de 1649 - et donc en période de crise politique à l'échelle européenne - son commerce a pu évoluer dans une zone grise qui nécessitait de laisser moins de traces. Cette hypothèse est renforcée par le fait qu'à la reprise de sa correspondance il entre en conflit avec des Lyonnais au sujet de ses mauvaises affaires de la décennie $1650^{67}$. Les années 1660 marquent un resserrement de ses relations autour d'un noyau

65. On sait par ailleurs qu'il est maître de la confrérie de Saint-Jean des merciers grossiers en 1639, de laquelle sont membres Jacques et Thomas de La Fosse dès 1629 (Arch. dép. de Seine-Maritime, 1 ER 2057, Lettres d'un marchand de Rouen, sans foliotation et 5 EP 527, Confrairie de Saint-Jean des merciers, grossiers, dépenses des années 1629 et 1639).

66. Ce registre représente près de 300000 caractères (espaces non compris), ce qui correspond à environ 70000 mots (Arch. dép. de Seine-Maritime, 1 ER 2057, Lettres d'un marchand de Rouen, sans foliotation).

67. Arch. dép. de Seine-Maritime, 1 ER 2057, sans foliotation, Lettres d'un marchand de Rouen, lettre à Aubin, 13 septembre 1665 . 
familial plus fermé, constitué de ses cousins. Ainsi, si son réseau se compose de quatre-vingt-cinq personnes pour les années 1647 et 1648, une dizaine d'années plus tard, il a profondément renouvelé son entourage. Il ne s'appuie alors que sur quatre-vingts individus pour une période six fois plus longue. Il n'écrit plus qu'à treize correspondants différents dans la décennie 1660 pour dix-sept précédemment. Sa correspondance est aussi proportionnellement moins longue envers ceux qui ne sont pas membres de sa famille, de l'ordre d'une fois et demie de caractères en moins, en moyenne. Robert de La Fosse organise donc essentiellement ses affaires autour de sa famille, dont son frère, Louis, aussi rouennais et associé à son négoce, qui s'est quant à lui spécialisé dans le commerce du blé jusqu'à son décès en 1667. Il compte aussi intensément sur ses cousins Thomas et Jacques de La Fosse, ainsi qu'un Blondel, aussi marchand, mais installé à Paris. Les activités marchandes de Robert s'orientent dès 1647 vers les Pays-Bas, Londres et l'Espagne ${ }^{68}$. L'étude de sa correspondance permet de cerner en détail la manière dont ce marchand à l'international organise ses affaires, gère son réseau et l'importance des flux de marchandises. L'idée étant aussi de s'interroger sur la place qu'occupe le commerce vers SaintMalo pour un marchand rouennais de son envergure.

Comme en témoigne ce diagramme ${ }^{69}$, qui représente le réseau commercial en lien avec Saint-Malo de Robert de La Fosse, sa correspondance semble montrer qu'il est le seul à pouvoir s'appuyer, en Bretagne, sur François Gaillard ${ }^{70}$. Ce dernier est issu d'une famille attestée à Saint-Malo depuis le début du Xvi ${ }^{\mathrm{e}}$ siècle. Les liens - exclusivement commerciaux - avec ce Malouin remontent probablement à la décennie 1650 et son association pousse le Rouennais à lui envoyer une dizaine de lettres et des marchandises par voie terrestre ou par mer ${ }^{71}$. Le réseau malouin concerne un nombre relativement réduit d'acteurs et Robert est le seul à être en contact direct avec ce port, ce qui le positionne indéniablement au cœur de ces échanges. Toutefois, il n'est pas en mesure d'entreprendre ce commerce sans l'appui de ses cousins et d'un certain nombre d'intermédiaires. De 1660 à 1664, il est en contact avec un marchand havrais nommé Broque qui fait indéniablement partie des éléments les plus importants, puisqu'il y est fait référence à onze reprises dans ses lettres. S'il est régulièrement associé à ses affaires, il joue

68. Arch. dép. de Seine-Maritime, 1 ER 2057, sans foliotation, Lettres d'un marchand de Rouen, lettre à Jeremie Moisson, 22 mars 1647, lettre à Richard Frenel, 23 mars 1647.

69. Réalisé par les soins de l'auteur à partir de la correspondance de Robert de La Fosse.

70. François Gaillard (1613-1687), sieur de Boisjoly et de La Motte. A été marchand de Saint-Malo et élu procureur syndic de la ville. En 1650, il épouse Françoise Le Breton (PARIS-JAllobert, Anciens registres paroissiaux de Bretagne, t. I : "Saint-Malo ", Rennes, 1898; LesPagnol, André, Messieurs de..., op. cit., p. 850).

71. En février 1661, Robert de La Fosse envoie par charrette deux tonneaux de marchandises " bien conditionnes " qui mettront huit jours à arriver. Augustin Demerelle (16261676) est voiturier de Rouen à Saint-Malo et n'a été utilisé qu'une seule fois par Robert afin de transporter des marchandises (Arch. dép. de Seine-Maritime, 3 E 00999, Registre paroissial de Saint-Vivien (1675-1677), registre 683, acte du 18 novembre 1676 et 1 ER 2057, Lettres d'un marchand de Rouen, lettre à Gaillard, 27 février 1661 et 13 mars 1661). 

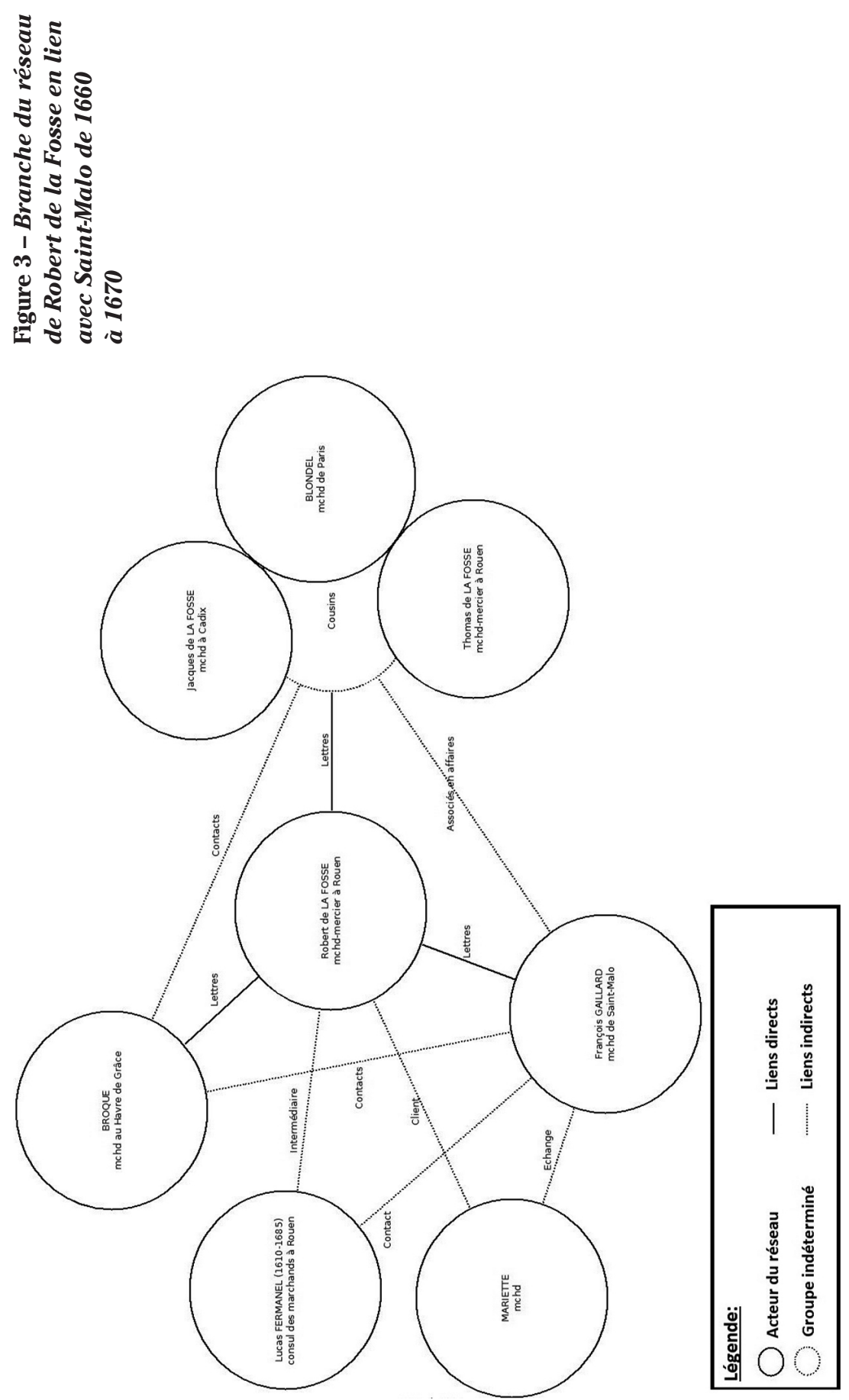
essentiellement le rôle d'avant-port pour l'importation de marchandises, en échange de commissions ${ }^{72}$. À partir de 1671, les deux marchands ne sont plus en contact puisque le marchand-mercier affirme que " jay un petit differen avec $\mathrm{M}^{\mathrm{r}}$ Broque du Havre ${ }^{73}$ ». Mariette est, lui aussi, impliqué dans les échanges malouins, mais son rôle est beaucoup plus limité. Ce marchand, que Robert connaît par l'intermédiaire d'autres acteurs de son réseau et qualifie de " tres honneste homme bien Capable \& qui a Bien estudié ${ }^{74}$ ", participe à des échanges de marchandises avec la famille de La Fosse à partir de 1662 et jusqu'à sa " disgrâce " de $1667^{75}$. Il est possible qu'il soit associé de manière privilégiée à l'un des cousins de Robert puisqu'il parle de " societé ${ }^{76}$ ", mais ce lien ne peut être défini plus précisément. Enfin, à un niveau plus local, Robert sait s'appuyer sur l'un des juges consuls des marchands de Rouen, Lucas Fermanel ${ }^{77}$. Lié à la famille de La Fosse dès 1660, celui-ci multiplie les conseils et participe à l'activité commerciale de Robert avec lequel il a de nombreuses rencontres. Toutefois, à partir 1668, un des cousins, probablement Jacques, entre en procès contre Fermanel et, selon Robert, cette situation lui cause " un grand tort " et l'embarrasse à tel point qu'il " avoit paine a se relever ${ }^{78}$ ". S'il réprimande d'abord son parent pour son attitude, Robert évolue progressivement dans son jugement vis-à-vis de Fermanel en considérant que " cest un ho[mm]e si froid ${ }^{79}$ " et semble mettre un terme à sa relation avec lui. Ainsi, le réseau malouin est composé d'un petit groupe de marchands interconnectés à travers un réseau peu cloisonné. Tous se connaissent, interagissent en s'échangeant des lettres, des informations et participent activement à la mise en place d'un commerce passant par SaintMalo. Néanmoins, ce réseau reste peu étoffé du point de vue des effectifs. Ainsi, de 1660 à 1672, Robert de La Fosse évoque au total près de quatrevingt-dix individus avec lesquels il entretient des relations, qu'il s'agisse des

72. Arch. dép. de Seine-Maritime, 1 ER 2057, Lettres d'un marchand de Rouen, lettre à Broque, novembre ou décembre 1660.

73. Arch. dép. de Seine-Maritime, 1 ER 2057, Lettres d'un marchand de Rouen, lettre à son cousin, juillet 1671 .

74. Arch. dép. de Seine-Maritime, 1 ER 2057, Lettres d'un marchand de Rouen, lettre à son cousin, 16 mai 1662.

75. Arch. dép. de Seine-Maritime, 1 ER 2057, Lettres d'un marchand de Rouen, lettre à son cousin, mars 1667.

76. Arch. dép. de Seine-Maritime, 1 ER 2057, Lettres d'un marchand de Rouen, lettre à son cousin, 16 mai 1662.

77. Lucas Fermanel (1610-1685), sieur de l'Epinay a été conseiller échevin et juge des consuls des marchands. Il habitait rue des Cordeliers et pourrait être l'un des correspondants de Colbert à Rouen en 1669. Il est régulièrement en affaire avec les Pays-Bas et l'Espagne (Arch. dép. de Seine-Maritime, 2 E 1 2525, acte du 20 mars 1649; 2 E 12316 , acte du 14 août 1660 et GIRARD, Albert, Le commerce français au temps des HabsbourgContribution à l'étude du commerce étranger en Espagne aux XVI et XVII siècles, Bordeaux, J. Bière, 1932, p. 139, 148 et 477).

78. Arch. dép. de Seine-Maritime, 1 ER 2057, Lettres d'un marchand de Rouen, lettre à son cousin, 25 février 1668 et 9 juin 1668.

79. Arch. dép. de Seine-Maritime, 1 ER 2057, Lettres d'un marchand de Rouen, lettre à son cousin, juillet 1671 . 
Figure 4 - Flux des échanges d'après la correspondance de Robert de La Fosse de 1647 à 1671

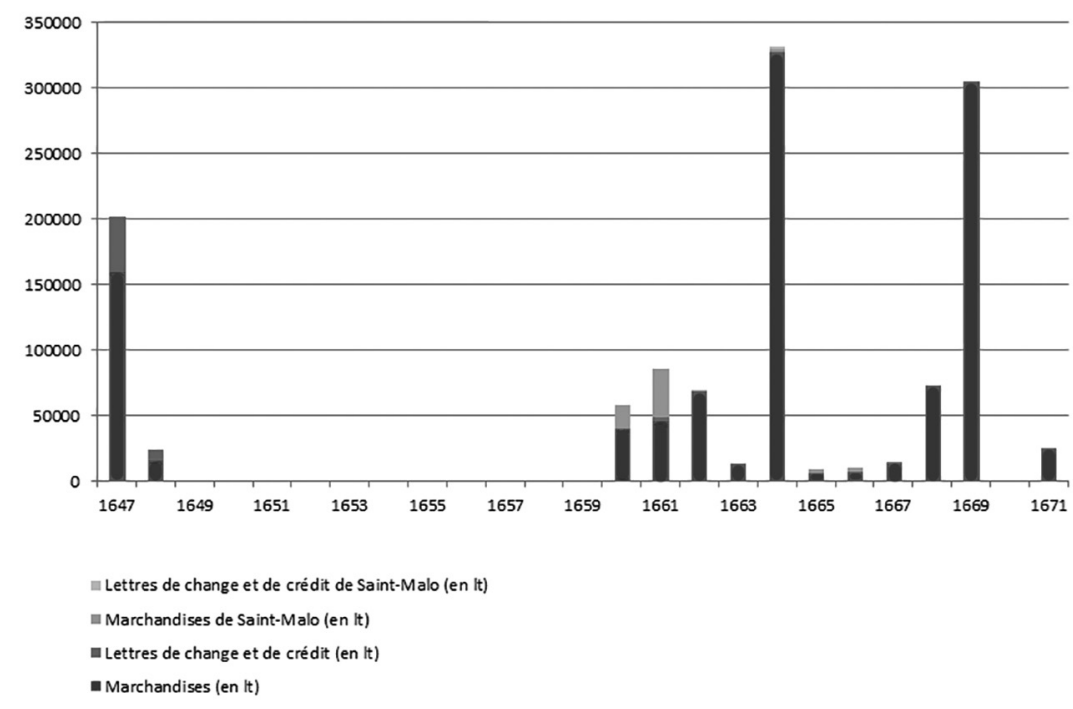

capitaines de navire, de marchands, de clients ou d'intermédiaires. Toutefois, si l'on peut juger l'importance d'un commerce par les flux de capitaux, SaintMalo constitue un élément d'attractivité au début de la décennie 1660.

Comme le montre ce graphique des flux monétaires et de marchandises de Robert de La Fosse ${ }^{80}$, celui-ci évoque en moyenne 44000 livres tournois d'échanges annuels de 1647 à 1671. Les lettres de change et de crédit ne représentent quant à elles que 2500 livres. Sur l'ensemble de la période étudiée, la part de Saint-Malo dans le flux d'échanges de ce marchand mercier apparaît comme relativement faible avec seulement 2700 livres annuels. Néanmoins, en prenant en compte uniquement la décennie 1660, touchant spécifiquement ce commerce, près de 6500 livres tournois sont échangées chaque année entre Rouen et Saint-Malo. En 1661, la part du commerce malouin représente plus de 20 \% des échanges de Robert de La Fosse et près de 40 \% en 1665, mais il s'agit d'années où les flux d'échanges de ce marchand mercier apparaissent comme relativement faibles, voire anecdotiques. Les années 1660 et 1666 dépassent elles aussi en moyenne le seuil des $40 \%$. L'orientation malouine du commerce de ce marchand est donc relativement explicite. Toutefois, cette analyse des

80. Réalisé par les soins de l'auteur à partir de la correspondance de Robert de La Fosse. Ce graphique prend en compte toutes les évocations monétaires - minimum dans le cas où plusieurs sont données - par ce marchand-mercier, qu'il s'agisse de livres tournois, d'écus ou de livres sterling. Pour l'ensemble de la période étudiée, le taux de change appliqué pour l'Angleterre est le seul qu'il annonce pour l'année 1648, c'est-à-dire 1 ई correspondant à 13,77 livres tournois. Par ailleurs, le prix de revente des marchandises n'est pas pris en compte dans ce recensement. Les assurances de navires s'intègrent dans la catégorie des "marchandises". 
flux ne paraît pas totalement satisfaisante, dans le sens où cette source ne peut être considérée de la même manière qu'un livre de comptes sériel. Étant donné qu'il s'agit d'une copie de correspondances, nous sommes tributaires de ce que l'auteur est disposé à transcrire; or de nombreux éléments laissent à penser qu'il n'a pas copié l'ensemble des lettres qu'il a envoyées durant sa carrière. Ce type de source offre, par ailleurs, d'autres perspectives. À travers cette correspondance, se dessinent les flux de navires mobilisés pour ces échanges. Ainsi, durant l'ensemble de la période, ce marchand mobilise une cinquantaine de navires pour transporter ses marchandises. Le malouin La Motte Gaillard est l'expéditeur de sept vaisseaux, principalement entre 1661 et 1666. Saint-Malo est aussi le port d'attache pour huit autres envois, à bord de navires tels que le Sainte-Hélène, le Saint-Jean-Baptiste ${ }^{81}$ ou La Palme, qui affrontent des conditions climatiques difficiles en juillet $1661^{82}$. Cette ville est aussi destinataire directe de huit cargaisons entre 1660 et 1661. À ce titre, le port breton est impliqué dans près de la moitié des navires utilisés par Robert de La Fosse ou ses partenaires, ce qui en fait indéniablement un rouage essentiel de ses échanges au début des années 1660. Entre ces deux grands ports, les marchandises échangées sont d'une grande diversité puisque l'on trouve de la quincaillerie, des boutons de cuir d'Espagne ou de Bruxelles, des jambettes $^{83}$, des chapeaux ou encore de la vigogne ${ }^{84}$. Le tout est transporté dans les tonneaux, ballots ou balles dont le conditionnement peut à l'occasion être problématique. C'est le cas en mars 1661, lorsque La Motte Gaillard reçoit « cinq [petites] bales de quincaillerie qui avoent este mouilles " ou lors de l'échouage d'un navire près de Quillebeuf, obligeant Robert de La Fosse à renvoyer les marchandises pour les faire " racommoder " ${ }^{85}$. Ainsi, en hiver, s'il faut une vingtaine de jours pour se rendre de Rouen à Saint-Malo par heu ${ }^{86}$ ou par charrette, les accidents sont fréquents ${ }^{87}$.

81. Le Saint-Jean-Baptiste jauge 300 tonneaux. Ce navire est équipé de 22 pièces de canon et a un équipage de 30 hommes. En 1649, son capitaine est le Hambourgeois Crestien Stubbe (Arch. dép. de Seine-Maritime, 2 E 1 2525, actes du 5 juin 1649).

82. Arch. dép. de Seine-Maritime, 1 ER 2057, Lettres d'un marchand de Rouen, lettre à son cousin, 2 juillet 1661 .

83. "Petit couteau pliant " (Dictionnaire de l'Académie Française, Paris, Première Édition, 1694, p. 579).

84. " Espece de mouton dont la laine sert à faire des chapeaux. Il signifie aussi la laine de cet animal " (Dictionnaire de l'Académie Française, op. cit., p. 643).

85. Arch. dép. de Seine-Maritime, 1 ER 2057, Lettres d'un marchand de Rouen, lettre à son cousin, 12 mars 1661.

86. Utilisés en Normandie et en Picardie, les heux sont des caboteurs de 50 à 100 tonneaux à fond plat et avec un seul mât. Ils servent principalement pour le passage de la Seine de Rouen au Havre-de-Grâce puisque peu de navires sont à même d'effectuer cette remontée (RICHARD, Robert, « Rouen, carrefour commercial entre les Flandres et l'Espagne au début du XVII ${ }^{\mathrm{e}}$ siècle ", dans Circuits commerciaux, foires et marchés en Normandie, $\mathrm{IX}^{\mathrm{e}}$ congrès des sociétés historiques, archéologiques et ethnologiques de Normandie, Rouen, CRDP, 1974, p. 12 et Nouveau Glossaire Nautique, Lettre H, Paris, CNRS Éditions, 1992, p. 902-904).

87. À titre d'exemple, une partie de la marchandise d'un navire est brûlée accidentellement à Cadix en 1662 (Arch. dép. de Seine-Maritime, 1 ER 2057, Lettres d'un marchand de Rouen, lettre à son cousin, 26 juin 1660, 30 octobre 1660, 27 novembre 1660). 
L'ensemble de ces données ne doit pas dissimuler le fait que le mode de transport n'est connu que pour une part infime des échanges de Robert de La Fosse, c'est-à-dire à peine 11000 livres sur la totalité de la période ${ }^{88}$. Toutefois, à travers l'étude de ce réseau, Saint-Malo apparaît comme un port de transit où certaines marchandises sont déchargées avant d'être embarquées à nouveau à destination de Rouen dans des navires plus petits permettant de remonter la Seine. Enfin, la correspondance montre que c'est une place essentielle dans le commerce vers l'Espagne puisque nombre de marchandises partent ou arrivent de Saint-Malo avant de rejoindre Jacques, un des cousins de Robert, installé à Cadix.

\section{Saint-Malo sur la route internationale}

La fin du XVI ${ }^{\mathrm{e}}$ siècle marque un profond bouleversement et la déstabilisation du système hérité du Moyen Âge. Ainsi, selon Guy Saupin, trois grandes nouveautés viennent bouleverser la domination castillane sur les échanges commerciaux : la révolte des Pays-Bas suivie de " la guerre de 80 ans ", la montée en puissance des colonies et le déclenchement de la guerre francoespagnole en $1635^{89}$. À partir de la fin du XVI siècle, les Malouins cherchent à pénétrer les marchés ibériques afin de contrebalancer l'absence d'un vrai marché intérieur. En parallèle, à la fin du XvII ${ }^{\mathrm{e}}$ siècle, la marine normande de commerce et de pêche correspond à 15 \% de la flotte marchande française, ce qui la place bien loin derrière la Bretagne ${ }^{90}$. L'implication rouennaise s'appuie sur l'importante communauté étrangère installée dans cette métropole. Pour le $\mathrm{XVII}^{\mathrm{e}}$ siècle, Jacques Bottin et André Lespagnol voient dans l'association bretonne et normande dans le commerce international à destination de la Méditerranée la mise en place d'un véritable " duopole". Il s'agit pour ces marchands de se partager la gestion de ce trafic international et cela même si les maisons rouennaises et malouines étaient en concurrence. On peut donc interpréter ces associations comme une logique de services croisés entre négociants des deux places ${ }^{91}$.

Ainsi, dans les échanges de Robert de La Fosse, on constate que ceux entre Saint-Malo et Cadix sont réguliers. De 1660 à 1669, une dizaine de transports de marchandises par navire impliquent ces deux espaces géo-

88. De 1647 à 1672, l'ensemble des flux monétaires évoqués par Robert de La Fosse représente plus d'un million de livres tournois.

89. PRIOTTI, Jean-Philippe et SAUPIN, Guy (dir.), Le commerce atlantique franco-espagnol Acteurs, négoces et ports ( $\mathrm{XV}^{\mathrm{e}-\mathrm{XVIII}}$ siècle), Rennes, Presses universitaires de Rennes, coll. "Histoire", 2008, pp. 9 et 12.

90. ZYSBERG, André, "Mesurer les activités au cabotage des navires normands à la fin du XVII ${ }^{\mathrm{e}}$ siècle ", Revue d'histoire maritime, $\mathrm{n}^{\circ} 8:$ : Histoire du cabotage européen aux XVI XIXe siècles ", Presses de l'université Paris-Sorbonne, 2008, p. 116.

91. Bottin, Jacques et LeSPAGNol André, " Un couple... ", art. cit., p. 43 et 55 et ETIENNE, Baptiste et GORET, Léa, « Dynasties marchandes, migrations et familles entre Rouen et le monde ibérique ", séminaire "Le temps de l'Empire ibérique (Xve-XvIII siècles) ", 25 septembre 2015, La forge numérique, [http://www.unicaen.fr/recherche/mrsh/forge/3734] (consulté le 28 décembre 2015). 
graphiques, alors que dans le même temps, il n'évoque qu'un départ de navire à destination de Londres. Durant une dizaine d'années, la valeur des affaires à destination de l'Espagne s'élève, en moyenne, à plus de 27000 livres tournois, signe s'il en est de l'importance de ce port breton dans le réseau à l'international du marchand rouennais. Toutefois, si au début du XVII ${ }^{\mathrm{e}}$ siècle ${ }^{92}$, aucun des partenaires ne vient à dominer l'autre, il semble qu'une soixante d'années plus tard les Rouennais prennent un réel ascendant sur le voisin breton. Saint-Malo semble alors faire office de port d'escale dans le cadre des échanges de Robert de La Fosse. Il s'agit donc d'une étape intermédiaire, mais essentielle pour le trafic à destination de l'Espagne. En outre, tous les correspondants de ce marchand mercier qui appartiennent au réseau malouin sont eux aussi en relation étroite avec le monde ibérique. S'il ne fallait qu'un exemple, en 1649, Lucas Fermanel utilise le navire nommé le Saint-Vincent, armé à Saint-Malo, pour envoyer cinq caisses d'indigo à Jean de Vinct, demeurant alors à Cadix ${ }^{93}$. En outre, les actes notariés concernant des Malouins montrent un fort attrait vers l'Espagne puisque $40 \%$ d'entre eux sont à destination de Cadix ou de Séville ${ }^{94}$. L'installation de marchands issus de Saint-Malo à Cadix est déjà bien connue et on comprend l'intérêt pour les marchands de Rouen d'utiliser ce réseau bien implanté ${ }^{95}$. Les Malouins y exportaient des toiles, dont une partie importante était fabriquée en Normandie, contribuant à l'importation de produits sur les marchés ibéro-américains durant tout le $\mathrm{XVII}^{\mathrm{e}}$ siècle $^{96}$. Des associations entre Rouennais et Malouins pour le commerce d'Espagne sont fréquentes. C'est le cas, par exemple, de Jean Richeome, sieur des Brosses, marchand de Saint-Malo qui cherche à recouvrer 10000 livres appartenant à Pierre Caramone, marchand français implanté à Séville. Depuis le début de la guerre entre la France et l'Espagne en 1635, cette somme était en possession des héritiers du marchand rouennais Jacques Thorel ${ }^{97}$. En

92. BotTin, Jacques et LeSPAgnol, André, "Un couple... », art. cit., p. 46.

93. Arch. dép. de Seine-Maritime, 2 E 1 2525, acte du 20 mars 1649.

94. Cela représente près de $60 \%$ des actes de Malouins en lien avec des territoires étrangers.

95. Archivo Historico Provincial de Cádiz, CA 2513, Testamento, Carlos Bolier, Saint Malo, acte du 3 octobre 1647, fo 101; CA 3030, Testamento, Pedro Harnon, Saint-Malo, acte du 22 mars 1625, fo 107; CA 3031, Testamento, Frederique Dominico, Saint-Malo, acte du 25 mai 1626, fo 276; Lespagnol, André, Messieurs de..., op. cit., p. 196 et BotTin, Jacques et LeSPAgnol, André, "Un couple... ", art. cit., p. 49.

96. André Lespagnol estime que les toiles fabriquées à Rouen constituent 36,5 \% de la ventilation (LesPaGnOL, André, Messieurs de..., op. cit., p. 429; MARTIN, Jean, "Les toiles bretagnes dans le commerce franco-espagnol de 1550 à 1830 ", Annales de Bretagne et des Pays de l'Ouest, t. 119, n ${ }^{\circ}$ 1, 2012, p. 31-60 et BotTin, Jacques, " De la toile au change : l'entrepôt rouennais et le commerce de Séville au début de l'époque moderne ", Annales du Midi : revue archéologique de la France méridionale, t. 117, n 251, 2005, p. 323-345).

97. Il pourrait s'agir d'un des deux Jacques Thorel décédés respectivement en 1650 et 1654 à Rouen, paroisse Saint-Maclou. Par ailleurs, on sait que ce marchand avait de son vivant une servante nommée Catherine Petit (Arch. dép. de Seine-Maritime, 3 E 00999, Registre paroissial de Saint-Maclou (1640-1651), registres 406-407, registre des sépul- 
1649, le marchand Jean Part ${ }^{98}$ s'appuie sur Nicolas Marcq, lui aussi marchand, mais d'origine anglaise et installé à Saint-Malo, pour retirer des marchandises du navire le Saint-Bergis, dont le maître est le Malouin Georges Raffin ${ }^{99}$. Nombre d'observateurs du temps ont mis en avant ce lien étroit entre l'Espagne, la Bretagne et la Normandie, pointant du doigt le déséquilibre de ce commerce :

"Les espagnols Jouissent en nostre païs et sur les marchandises de nostre creu a nostre grand preiudice; et convient bien remarquer que cela n'est pas de petite Importance. Car les Espagnols traffiquans aux Indes font charger à Roüen, S[ain]t Malo et autres endroits de La Bretagne et Güienne autant de marchandises que La France en envoyé en espagne, et pour combler de Leur artiffice, ils se bornent ordinairem[ent] en espagne à n'achepter Les marchandises des forains, sinon qua Lextremité ${ }^{100}$."

Les marchands rouennais dénoncent ainsi que ce commerce "sort[e] de la main de La France et tombe en celle des estrangers ", profitant des réserves d'or inépuisables des colonies ${ }^{101}$. Or, dans le cas de Robert de La Fosse et des autres marchands français impliqués dans le commerce avec le royaume d'Espagne, ceux-ci semblent prendre en main ce trafic ou du moins en être à l'initiative. Néanmoins, l'orientation du port de Saint-Malo comme plaque tournante des affaires rouennaises ne l'est pas uniquement à destination de l'Espagne. Si on observe les témoins rouennais des actes notariés impliqués dans le commerce entre Saint-Malo, Cadix et Séville, l'écrasante majorité d'entre eux se retrouvent aussi dans des actes qui impliquent des Bretons en lien avec la Hollande, l'Angleterre et, dans une moindre mesure, le Portugal. Ainsi, Jacques Gilles ${ }^{102}$, qui est témoin dans un acte en 1649 touchant Saint-Malo et Cadix, l'est à nouveau plus de deux mois plus tard avec un acte concernant cette fois Hambourg ${ }^{103}$. En 1649, Pierre Adam ${ }^{104}$ est le témoin d'un acte passé par le marchand

tures, acte du 18 septembre 1650; 3 E 00999, Registre paroissial de Saint-Maclou (16541660), registre 408, registre des sépultures, acte du 30 juillet 1654 et 2 E 12491 , acte du 19 octobre 1660 et 2 E 12491 , acte du 30 juillet 1660$)$.

98. Jean Part ou Parr (1601-1651), marchand protestant, demeurant à Rouen et d'origine anglaise. Il est très impliqué dans le commerce avec Londres et Pierre Adam est régulièrement témoin de ses actes notariés (Arch. dép. de Seine-Maritime, 4 E 03395, Registre du temple protestant de Quevilly (1643-1659), registre des sépultures, acte du 15 décembre 1651; 2 E 1 2387, acte du 3 mars 1649; 2 E 1 2390, acte du 15 octobre 1649; 2 E 1 2388, actes du 27 avril 1649, 25 mai 1649 et 21 juin 1649 et 2 E 1 2391, acte du 2 mars 1650).

99. Arch. dép. de Seine-Maritime, 2 E 1 2390, acte du 15 octobre 1649.

100. BnF, Cinq cents de Colbert 203, " Mémoire et advis donnes au Roy sur Les fait de la navigation et commerce de L'Amerique et Les ordres des armées d'Espagne ", fo 164. 101. Idem.

102. Jacques Gilles (?-1653) est décédé et enterré catholiquement paroisse Saint-Godard (Arch. dép. de Seine-Maritime, 3 E 00999, Registre paroissial de Saint-Godard (1629-1667), registre 185, registre des décès, acte du 28 juin 1653).

103. Arch. dép. de Seine-Maritime, 2 E 1 2525, acte du 20 mars 1649 et 5 juin 1649.

104. Il pourrait s'agir de Pierre Adam (1604-1679), passementier protestant. Marié en 1668 à Jeanne Jolly. À lui seul, Pierre est témoin dans plus de $23 \%$ des actes passés à Rouen et concernant des Bretons (Arch. dép. de Seine-Maritime, 4 E 03401, Registre du temple 
rouennais Guillaume $\operatorname{Scot}^{105}$ : celui-ci cherche à charger Nicolas Éon ${ }^{106}$, marchand de Saint-Malo, de recevoir du procureur fiscal de l'argent de Charles Tranchant ${ }^{107}$, envoyé pour sa femme décédée alors qu'elle était installée en Espagne. La même année, il est à nouveau témoin d'un acte concernant un Breton en lien avec l'Angleterre et Rotterdam puis, en 1650, de deux autres actes touchant à nouveau la Hollande ${ }^{108}$. Par ailleurs, les acteurs mêmes des actes notariés à l'égard des Malouins renvoient, à l'occasion, à l'Angleterre ou Hambourg. Ainsi, deux actes concernent Londres, à l'image de celui impliquant le futaillier Nicolas Helot ${ }^{109}$ présentant à un des frères du marchand malouin Anthoine Thorin ${ }^{110}$, alors installé à Rouen, une lettre de change rédigé à Londres et d'une valeur de 800 livres tournois. En fin de journée, celle-ci est finalement refusée par le marchand de Saint-Malo et son associé ${ }^{111}$. On a donc, dans ces cas précis, la mise en place de véritables réseaux internationaux reposant sur plusieurs débouchés européens majeurs.

protestant de Quevilly (1676-1680), registre des sépultures, acte du 16 août 1679et Château du Bois-Tiffrais, Fiches des protestants normands, par Denis Vatinel, 22 A 1).

105. Guillaume Scot, marchand est très impliqué dans le commerce avec les Pays-Bas avec des intermédiaires d'origine ibérique (Arch. dép. de Seine-Maritime, 2 E 1 2491, actes du 4 septembre 1660, 24 septembre 1660, 10 octobre 1660 et 2 E 12490 , actes du 12 janvier 1660, 25 février 1660, 18 février 1660).

106. Nicolas Éon (1603-1668), sieur de La Baronnie, fils de Guillaume et de Jeanne Moreau. Il est issu d'une puissante famille installée à Saint-Malo depuis au moins le début du $\mathrm{XVI}^{\mathrm{e}}$ siècle. Cette famille est engagée de longue date dans le commerce international et est dispersée aux XVII ${ }^{\mathrm{e}}$ et XVIII ${ }^{\mathrm{e}}$ siècles à travers le territoire français. Il pourrait être impliqué dans un second acte notarié rouennais dans lequel il réclame, avec son frère Julien, au marchand rouennais Anthoine Asselin (?-1701) la somme de 1200 écus " a soixante solz pour Escu " sur une lettre de change d'Amsterdam. Dans les années 1680, la famille Éon disposait de correspondants à travers toute l'Europe et en particulier à Rouen (Arch. dép. de Seine-Maritime, 2 E 1 2490, acte du $1^{\mathrm{er}}$ avril 1660; 3 E 00999, Registre paroissial de SaintVincent (1700-1704), registre 639, acte du 18 juillet 1701 et LESPAGNOL, André, Messieurs de..., op. cit., p. 165 et 849$)$.

107. Charles Tranchant, marchand de Saint-Malo, est aussi impliqué dans un autre acte avec les frères Robert et Louis Le Francois (1607-1667 et 1620-1682), marchands de Rouen, visant à recouvrer des marchandises se trouvant à Séville entre les mains des héritiers du marchand Jean Viel (Arch. dép. de Seine-Maritime, 2 E 1 2392, acte du 10 juin 1650; 3 E 00999, Registre paroissial de Saint-Denis (1647-1667), acte du 3 avril 1667 et 3 E 00999 , Registre paroissial de Saint-Sauveur (1674-1692), acte du 13 avril 1682).

108. Arch. dép. de Seine-Maritime, 2 E 1 2387, acte du 19 janvier 1649; 2 E 1 2467, acte du 21 juillet 1649 et 2 E 12391 , actes du 4 février 1650 et 14 mars 1650 .

109. La famille des Helot compte de nombreux tonneliers, marchands-merciers et de futailliers. Les futailliers fabriquent des futailles, c'est-à-dire des "vaisseau(x) de bois à mettre du vin " ou des tonneaux (Dictionnaire de l'Académie Française, op. cit., p. 503).

110. Dans l'hypothèse où il pourrait être décédé à Rouen : Anthoine Thorin (1617-1674). Son frère est sans doute Jean Thorin (1605-1660), marchand à Rouen (Arch. dép. de SeineMaritime, 4 E 03398, Registre du temple protestant de Quevilly (1660-1668), registre des sépultures, acte du 191660 et 4 E 03401, Registre du temple protestant de Quevilly (1674), registre des sépultures, acte du 10 avril 1674).

111. Arch. dép. de Seine-Maritime, 2 E 1 2469, acte du 10 septembre 1650. 
«En ce temps ou le malheur veut que l'on ne negotie que sur des navires Empruntés, \& sous ce nom de nos alliéz, mesme pour faire le traffic des affaires de france les Unes avec les autres co[mm]e de Normandie En Bretagne, de Bretagne En Gascogne \& de ces lieux la L'on est obligé de se servir des Navires Hollandois \& Hambourgois, Et ce seroit une precaution bien Inutille \& tres dangereuse si on Estoit obligé de registrer les cognoissements \& les chartes parties ${ }^{112}$."

En février 1657, l'importance des liens entre la Bretagne, la Normandie et la Hollande en période de guerre est manifeste dans ce " mémoire " de marchands, copié par le conseiller Robert Bigot ${ }^{113}$ dans son manuscrit portant sur le parlement de Normandie. Il met en avant les revendications des marchands rouennais alors que les chambres sont assemblées pour lire l'interdiction d'utiliser des navires étrangers pour le cabotage au sein du royaume de France. Il semble que la nécessité du recours aux pavillons étrangers en période de guerre ait été reconnue par certains parlementaires puisque l'édit est finalement refusé à une courte majorité. Bigot justifie ce refus en affirmant que " lon Voioit grand nombre de famille considerables que la difficulté du Commerce avoit depuis quelques années extraordinairem[ent] Incommodé ${ }^{114}$ ". L'intermédiaire néerlandais et donc l'utilisation de pavillons étrangers pour le trafic d'Espagne est expliqué, par ailleurs, comme relevant d'une nécessité, au risque de se faire saisir des marchandises " contre l'equité naturelle ${ }^{115}$ ". Encore une fois, dans ces cas de figure, les marchands rouennais insistent sur les liens entre Normandie et Bretagne, mais aussi sur le rôle international de ces deux provinces et ils ne sont pas les seuls. À la fin du XVII ${ }^{\mathrm{e}}$ siècle, les Mémoires Touchant Le Commerce Que les provinces unies des païs bas fontz n'hésitent pas à associer la Bretagne et la Normandie pour comptabiliser le commerce des lingeries et des toiles vers la Hollande et proposent un total de cinq millions de livres tournois ${ }^{116}$. Enfin, de nombreux cas de mise en place de compagnies associant pour le commerce vers le Canada des Malouins et des Rouennais ont déjà fait l'objet d'études. C'est le cas notamment de celle fondée par De Monts, avec un capital de 90000 livres fournis par des marchands de Rouen, Saint-Malo, La Rochelle et Saint-Jean-de-Luz ou, encore, en 1613, avec la fondation d'une

112. Dans ce manuscrit, Robert Bigot recopie un « Mémoire pour le Commerce contre le pretendu Establissement de deux greffiers ou Nottaires dasseurance ", d'où est extrait cette citation (Bibliothèque municipale de Rouen, Montbret 986, Depuis la S[ain]t Martin 1657 Jusques a la S[ain]t Martin 1658, par Robert Bigot, fo 8).

113. Robert Bigot (1633-1692), baron de Monville. A été avocat puis conseiller au parlement de Rouen en 1654 et, enfin, conseiller au parlement de Paris. En février 1655, il a épousé Marie Du Moucel, dame de Sassetot (FrondEVILLE Henri et Odette de, Les conseillers du Parlement de Normandie de 1641 à 1715 - Recueil généalogique établi sur la base du Manuscrit Bigot de la Bibliothèque de Rouen, t. Iv, Rouen, Lestringant, 1970, p. 292).

114. Bib. mun. de Rouen, Montbret 986, Depuis la S[ain]t Martin 1657 Jusques a la S[ain] t Martin 1658, par Robert Bigot, fo 7 .

115. BnF, Cinq cents Colbert 203, "Memoires, Pour les Marchands de Paris, Roüen, S. Malo, Nantes, Morlaiz, le Havre, \& Dieppe, Trafiquans en Mer », p. 3.

116. Bib. mun. de Rouen, coll. Montbret, ms 225, "Memoires touchant le commerce que les Provinces Unies des Païs Bas font dans les divers endroits du monde ", 1694, fo 57. 
société - moyennant une redevance annuelle de 1000 écus - pour le monopole de la région supérieure de la vallée du Saint-Laurent ${ }^{117}$.

L'association entre les provinces de Bretagne et de Normandie et entre les villes de Saint-Malo et de Rouen dépasse donc largement le cadre du mythe politique, en particulier du point de vue du commerce. Ces trajectoires différentes ne paraissent pas avoir d'impact sur les échanges entre les deux provinces. Comme l'a prouvé Jacques Bottin, ces liens étaient intenses dans la première moitié du XVII ${ }^{\mathrm{e}}$ siècle ${ }^{118}$, ils le sont restés durant la Fronde et semblent même se renforcer durant les décennies suivantes, comme en témoigne la correspondance de Robert de La Fosse. Durant les années 1660, ce marchand mercier a su placer Saint-Malo au cœur de son activité marchande à destination de l'Espagne, en en faisant l'avant-port de Rouen grâce à un réseau restreint, mais interconnecté. Les affaires commerciales entre ces deux villes sont parfois florissantes, composées d'associations économiques et politiques, mais elles peuvent aussi déboucher sur des conflits à l'image du procès engagé par Nicolas Bauquemare. Ces liens commerciaux sont aussi et surtout une porte d'entrée pour les Rouennais vers un commerce international à destination de la Nouvelle France, des Indes, des Pays-Bas et plus marginalement de l'Angleterre. Nombre de marchands de Rouen privilégient des associations avec des marchands malouins, plutôt que ceux d'autres ports normands, comme Dieppe ou le Havre-de-Grâce, qui pourraient remplir les mêmes fonctions. C'est notamment le cas du port dieppois dont Robert de La Fosse se sert pour la mise en place d'échanges avec l'Angleterre à la fin des années 1640. Tout laisse à penser qu'il s'agit pour ces marchands de saisir des opportunités, à l'image des La Fosse qui s'appuient sur le malouin François Gaillard pour développer leurs affaires en Espagne à travers une relation de longue date. Cette association n'est qu'une parenthèse pour Robert de La Fosse qui, durant vingt-cinq années, se saisit des meilleures occasions et multiplie les aventures commerciales, parfois risquées.

Rouen et Saint-Malo sont donc deux villes à vocation commerciale, mais de dimension et d'importance incomparable au milieu du XVII ${ }^{\mathrm{e}}$ siècle. Ces deux ports présentent de surcroît des trajectoires politiques et économiques aux antipodes durant les révoltes des Nu-pieds et celle de la Fronde. Ainsi, à partir de 1650, Rouen souffre des guerres de Louis XIV et se dépeuple de ses marchands, en raison des départs des Hollandais et de certains protestants ${ }^{119}$. Pourtant, oscillant entre espoirs et désillusions, l'autorité royale cherche à unifier ces deux espaces en un ensemble poli-

117. Lacoursière, Jacques, Provencher, Jean et VAugeors, Denis, Canada-Québec (15342000), Sillery, Éditions du Septentrion, 2001, p. 36 et 45 et MATHIEU, Jacques, La NouvelleFrance - Les Français en Amérique du Nord (XVI'-XVIII siècle), Québec et Paris, Presses de l'université de Laval et Belin, 1991, p. 102.

118. Bottin, Jacques et LesPagnol, André, « Un couple... », art. cit., p. 50-57.

119. Chaline, Olivier, "La société... ", art. cit., p. 42. 
tique et économique cohérent afin de se préserver de la «fureur des normans ${ }^{120}$ ". Cette volonté reste lettre morte puisqu'on observe bien peu de relations au quotidien entre ces deux places côtières voisines et s'ignorant pratiquement. Il y a peu de Malouins à Rouen, peu d'implication rouennaise à Saint-Malo. On explore donc en vain les sources normandes afin de trouver des traces de liens entre ces deux villes et provinces dos à dos.

\section{RÉSUMÉ}

Rouen et Saint-Malo sont indéniablement de taille et d'échelle différentes durant l'époque moderne. Or, ces deux villes marchandes entretiennent des liens étroits au cour du XVII ${ }^{\mathrm{e}}$ siècle. Les liens politiques qui les unissent apparaissent dans les sources. Néanmoins, la situation entre ces deux provinces voisines semble opposée. Comme durant la révolte des Nu-pieds, une dizaine d'années auparavant, la Normandie s'engage dans la Fronde alors que la Bretagne demeure calme. Les liens commerciaux sont quant à eux omniprésents entre ces deux ports. Se rencontrent des alliances parfois et, surtout, des conflits et des procès importants. Le cas de Robert de La Fosse, un marchand mercier de Rouen, est intéressant. Celui-ci fait de Saint-Malo et de ses relations d'affaires sur place un relais important de son trafic international et, en particulier, pour les trafics espagnols.

\section{ABSTRACT}

Rouen and Saint Malo were two cities of very different sizes in the modern age. Yet these two commercial cities had close ties throughout the $17^{\text {th }}$ century. Sources show political connections between the two, but their situations were nevertheless completely different. Normandy took part in the Fronde, as it did ten years before during the Revolt of the va-nu-pieds, whereas Brittany remained quiet. Commercial links were maintained between these two ports. There were some agreements put in place but connections were dominated by conflicts and important trials. The case Robert de La Fosse, a marchand-mercier of Rouen, is of particularly interest. He puts Saint-Malo at the core of his international business, especially with Spain.

120. La Fureur des Normans contre les Mazarinistes, Pierre Variquet, Paris, 1649. 